\title{
On the global well-posedness of the 3D axisymmetric resistive MHD equations
}

\author{
Zineb Hassainia
}

\begin{abstract}
In this paper, we prove the global well-posedness for the three-dimensional magnetohydrodynamics (MHD) equations with zero viscosity and axisymmetric initial data. First, we analyze the problem corresponding to the Sobolev regularities $H^{s} \times H^{s-2}$, with $s>5 / 2$. Second, we address the same problem but for the Besov critical regularities $B_{p, 1}^{3 / p+1} \times B_{1, p}^{3 / p-1}, 2 \leqslant p \leqslant \infty$. This case turns out to be more subtle as the Beale-Kato-Majda criterion is not known to be valid for rough regularities.
\end{abstract}

\section{Contents}

\section{Introduction}

\section{Tools and function spaces}

3 Axisymmetric flows

4 A priori estimates

5 Sub-critical regularities $\quad 18$

5.1 Lipschitz bound of the velocity . . . . . . . . . . . . . . . 18

5.2 Existence . . . . . . . . . . . . . . . . . . . . . . . 21

5.3 Uniqueness result . . . . . . . . . . . . . . . . . . . . . 21

6 Critical regularities $\quad 22$

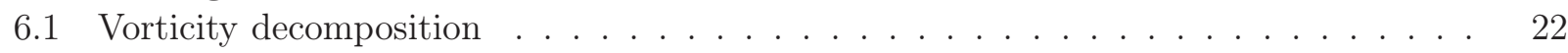

6.2 Lipschitz bound . . . . . . . . . . . . . . . . . . . . 25

\begin{tabular}{ll} 
A Appendix & 29 \\
\hline
\end{tabular}

\section{Introduction}

In this paper, we consider the three-dimensional incompressible magnetohydrodynamic (MHD) system, describing the motion of an electrically conducting fluid with a neglected viscosity and an important resistivity,

$$
\left\{\begin{array}{l}
\partial_{t} v+v \cdot \nabla v+\nabla p=b \cdot \nabla b, \quad t \in \mathbb{R}_{+}, x \in \mathbb{R}^{3} \\
\partial_{t} b+v \cdot \nabla b-\Delta b=b \cdot \nabla v \\
\operatorname{div} v=0, \operatorname{div} b=0
\end{array}\right.
$$


where $v$ denotes the fluid velocity and $b$ stands for the magnetic field. The pressure $p$ is a scalar function that can be recovered from the velocity and the magnetic field by inverting an elliptic equation. Note that we have set the magnetic diffusion coefficient to be one by a scaling transformation. For a general review about the derivation of the MHD equations we refer the reader to [6, 13, 19].

Since the pioneering work of Alfvén [2], the MHD equations have played prominent roles in the study of significant phenomena such as the magnetic reconnection in astrophysics, geomagnetic dynamo in geophysics and plasma confinement in engineering, see [6, 19]. From mathematical point of view, the MHD system and its ideal, fully dissipative and partially dissipative counterparts have been intensively investigated in the few last decades and considerable interest has been devoted to the local/global well-posedness problem, see for instance [20, 40, 9, 12, 16, 15, 10, 22, 23, 28, 35, 37, 38, 45] and the references therein. Note that the global existence and the uniqueness of such solutions is an open problem except in the two dimensional case with the full dissipation.

Hereafter, we shall primarily restrict the discussion to the MHD equations with only magnetic diffusion (1.1). In [30], Kozono proved global existence of weak solutions in 2D for initial data in $L^{2}$; while in 3D, Fan and Ozawa 21] showed that the solution can be extended beyond time $T$ if $\nabla v \in L^{1}\left(0, T ; L^{\infty}\left(\mathbb{R}^{3}\right)\right)$. However, it is not clear if such weak solutions are unique or if one can extend the the global weak theory, in $2 \mathrm{D}$, to the classical one. This latter problem is critical in the sense that if we replace the Laplacian term $-\Delta$ by a fractional Laplacian operator $(-\Delta)^{\beta}, \beta>1$, the resulting system then admits a unique global solution. This fact was proved independently in [11, 29]. We stress that the crucial part, to prove the global well posedness for (1.1) in $2 \mathrm{D}$, is to get an a priori estimate for the vorticity $\omega:=\operatorname{curl}(v)$ in $L^{\infty}$. The main difficulty can be illustrated through the equations governing the vorticity $\omega:=\operatorname{curl}(v)=\partial_{1} v_{2}-\partial_{2} v_{1}$ and the current density $j:=\operatorname{curl}(b)=\partial_{1} b_{2}-\partial_{2} b_{1}$,

$$
\left\{\begin{array}{l}
\partial_{t} \omega+v \cdot \nabla \omega=b \cdot \nabla j \\
\partial_{t} j+v \cdot \nabla j-\Delta j=b \cdot \nabla j+2 \partial_{1} b \cdot \nabla v_{2}-2 \partial_{2} b \cdot \nabla v_{1}
\end{array}\right.
$$

According to the first equation in the above system, the vorticity conservation requires a global bound of $\|\nabla j\|_{L \infty}$. However, the nonlinear structure of the term $\mathcal{Q}(\nabla v, \nabla b):=2 \partial_{1} b \cdot \nabla v_{2}-2 \partial_{2} b \cdot \nabla v_{1}$ and the lack of continuity of Riesz transforms on the bounded functions makes the the problem highly non trivial. For regularity criteria we refer the reader for instance to [9, 32, 29].

The main scope of this paper is to construct global unique classical solutions in 3D under some geometrical constraints. More precisely, we shall see that the axisymmetry offer a suitable class of initial data for which the construction of classical solutions is possible. Note that this has been successfully implemented for the viscous non-resistive MHD equations, by Lei in [31] where he proves the existence of axisymmetric global classical solutions of the special form

$$
v(t, x)=v^{r}(t, r, z) e_{r}+v^{3}(t, r, z) e_{z}, \quad b=b^{\theta}(t, r, z) e_{\theta}
$$

where $r=\left(x_{1}^{2}+x_{2^{2}}\right)^{\frac{1}{2}},\left(e_{r}, e_{\theta}, e_{z}\right)$ is the cylindrical basis of $\mathbb{R}^{3}$. We stress that the fluid velocity $v$ and the magnetic field $b$ are assumed to be invariant by rotation around the vertical axis.

Before stating our contribution in the subject let us briefly discuss what is known for Euler equations ( obtained by setting $b$ identically zero) with this special initial data. In [43], Ukhovskii and Yudovich proved the global existence for axisymmetric initial data with finite energy and satisfying in addition $\omega_{0} \in L^{2} \cap L^{\infty}$ and $\omega_{0} / r \in L^{2} \cap L^{\infty}$. This result has been improved by Shirota and Yanagisawa [42] who proved global existence in $H^{s}$, with $s>5 / 2$. In [17], Danchin has obtained global existence and uniqueness for initial data $\omega_{0} \in L^{3,1} \cap L^{\infty}$ and $\omega_{0} / r \in L^{3,1}$, where $L^{3,1}$ denotes the Lorentz space. The global well-posedness in the critical Besov regularity, that is, $v_{0} \in B_{p, 1}^{3 / p+1}$, 
$p \in[1, \infty]$ with $\omega_{0} / r \in L^{3,1}$ was established in [3]. We point out that the global well-posedness result has been extended for 3D Euler-Boussinesq system, which couples Euler equations with a transport-heat equation governing the density, see [27, 41.

Our main concern here is to validate similar global well-posedness results for the MHD system (1.1). Our first result reads as follows

Theorem 1.1. Let $v_{0}$ and $b_{0}$ be two axisymmetric divergence-free vector fields as in (1.3). Suppose that $\left(v_{0}, b_{0}\right) \in H^{s} \times H^{s-2}$, with $s>5 / 2$, and $b_{0}^{\theta} / r \in L^{2} \cap L^{\infty}$. Then there exists a unique global solution $(v, b)$ to the MHD system (1.1) such that

$$
(v, b) \in \mathcal{C}\left(\mathbb{R}_{+} ; H^{s}\right) \times\left(\mathcal{C}\left(\mathbb{R}_{+} ; H^{s-2}\right) \cap \widetilde{L}_{\mathrm{loc}}^{1}\left(\mathbb{R}_{+} ; H^{s}\right)\right) \quad \text { and } \quad \frac{b}{r} \in L_{\mathrm{loc}}^{\infty}\left(\mathbb{R}_{+} ; L^{2} \cap L^{\infty}\right) .
$$

Our second main result deals with the critical Besov spaces.

Theorem 1.2. Let $p \in[2, \infty], v_{0} \in L^{2} \cap B_{p, 1}^{3 / p+1}$ and $b_{0} \in L^{2}$ be two axisymmetric divergence-free vector fields as in (1.3). Assume, in addition, that $\omega_{0}^{\theta} / r \in L^{3,1}$ and $b_{0}^{\theta} / r \in L^{2} \cap L^{\infty}$, where $\omega_{0}^{\theta}$ is the angular component of the vorticity $\omega_{0}:=\operatorname{curl}\left(v_{0}\right)$.

- Case $p=\infty$. If $b_{0} \in B_{\sigma, 1}^{3 / \sigma-1}, \sigma \in[2, \infty)$, then there exists a unique global solution $(v, b)$ to the system (1.1) such that

$$
(v, b) \in \mathcal{C}\left(\mathbb{R}_{+} ; B_{\infty, 1}^{1}\right) \times\left(\mathcal{C}\left(\mathbb{R}_{+} ; B_{p, 1}^{3 / \sigma-1}\right) \cap L_{\mathrm{loc}}^{1}\left(\mathbb{R}_{+} ; B_{\infty, 1}^{1}\right)\right)
$$

- Case $p<\infty$. If $b_{0} \in B_{p, 1}^{3 / p-1}$ then there exists a unique global solution $(v, b)$ to the system (1.1) such that

$$
(v, b) \in \mathcal{C}\left(\mathbb{R}_{+} ; B_{p, 1}^{\frac{3}{p}+1}\right) \times\left(\mathcal{C}\left(\mathbb{R}_{+} ; B_{p, 1}^{3 / p-1}\right) \cap L_{\mathrm{loc}}^{1}\left(\mathbb{R}_{+} ; B_{p, 1}^{3 / p+1}\right)\right) .
$$

Moreover, in both cases, one has

$$
\left(\frac{\omega}{r}, \frac{b}{r}\right) \in L_{\mathrm{loc}}^{\infty}\left(\mathbb{R}_{+} ; L^{3,1}\right) \times L_{\mathrm{loc}}^{\infty}\left(\mathbb{R}_{+} ; L^{2} \cap L^{\infty}\right) .
$$

A few remarks are in order

\section{Remark 1.1.}

(i) The condition $\omega_{0} / r \in L^{3,1}$ is automatically derived from $v_{0} \in B_{p, 1}^{1+3 / p}$ for $p<3$, see (2.4).

(ii) For $p>2$ the assumption $v_{0} \in L^{2}$ is still needed because the only energy estimate owned for the MHD system is in $L^{2}$.

(iii) In the limiting case $p=\infty$, the critical space for magnetic field is $B_{\infty, 1}^{-1}$. However, we were unable to get smoothing effects on this space and had to relax with the assumption $b_{0} \in B_{\sigma, 1}^{3 / \sigma-1}$, for some $\sigma<\infty$.

(iv) Note that if $\sigma \in(3, \infty)$ then we have $b \in L_{\text {loc }}^{1}\left(\mathbb{R}_{+} ; B_{\sigma, 1}^{3 / \sigma+1}\right)$.

Now, we shall briefly discuss the main ideas of the proof. Note that, in space dimension three, the vorticity $\omega:=\operatorname{curl}(v)$ satisfies the equation

$$
\partial_{t} \omega+v \cdot \nabla \omega=\omega \cdot \nabla v+\operatorname{curl}(b \cdot \nabla b)
$$


and the way to control the vortex stretching term in the right-hand side is a widely open problem even for trivial magnetic fields. Nevertheless, for axisymmetric flows, as in (1.3), the vorticity has the special form

$$
\omega=\omega^{\theta} e_{\theta}
$$

and the equation (1.4) can be written as

$$
\partial_{t} \omega+v \cdot \nabla \omega=\frac{v^{r}}{r} \omega-\frac{\partial_{z}\left(b^{\theta}\right)^{2}}{r} e_{\theta} .
$$

Thus, by dividing the vorticity equation by $r$, one can absorb the vortex stretching term into the convection term, leaving only one term involving the quatity $\Gamma:=b^{\theta} / r$ as a forcing one. More precisely, the scalar function $\Omega:=\omega^{\theta} / r$ satisfies

$$
\partial_{t} \Omega+v \cdot \nabla \Omega=-\partial_{z} \Gamma^{2}
$$

In the light of the proof for the 3-D axisymmetric Euler equations, the key point is to get a control of the Lorentz norm $\|\Omega\|_{L^{3,1}}$. This, according to (1.5), requires some strong a priori estimates on $\Gamma$. Since the magnetic fields has the spacial form (1.3) then the magnetic stretching term in the second equation of (1.1) reads

$$
b \cdot \nabla v=\frac{v^{r}}{r} b^{\theta},
$$

and the quantity $\Gamma:=\frac{b^{\theta}}{r}$ solves the equation

$$
\partial_{t} \Gamma+v \cdot \nabla \Gamma-\left(\Delta+\frac{2}{r} \partial_{r}\right) \Gamma=0 .
$$

Therefore, by using the smoothing effect of the heat flow we obtain the desired control on $\Gamma$ and then on $\Omega$. This allows to get the $L^{\infty}$ bound of the vorticity $\omega$ for every time through the Biot-Savart law. Then, in order to globally propagate the optimal subcritical regularities, in the scaling sense, of the MHD system (1.1) we uses some refined informations about the axisymmetric geometry of the magnetic field $b$.

The situation is more complicate for the critical Besov regularities since the Beale-Kato-Majda criterion significant quantity that one should estimate is $\|\omega\|_{B_{\infty, 1}^{0}}$. For this aim we use the approach developed in [3] where the axisymmetric geometry plays a crucial role. This allows to bound for every time the Lipschitz norm of the velocity and then to propagate the regularities.

The rest of this paper is organized as follows. In section 2 , we recall some function spaces and give some of their useful properties, we also gather some preliminary estimates. In Section 3 we shall be concerned with some a priori estimate. In section 5 and 6 we give respectively the proof of Theorem 1.1 and Theorem 1.2. In the appendix, we establish some product laws for axisymmetric flows.

\section{Tools and function spaces}

Throughout this paper, $C$ stands for some real positive constant which may be different in each occurrence and $C_{0}$ for a positive constant depending on the size of the initial data. We shall sometimes alternatively use the notation $X \lesssim Y$ for an inequality of the type $X \leqslant C Y$. Also, for any pair of operator $D$ and $F$ on some Banach space $\mathcal{A}$, the commutator $[D, F]$ is defined by $D F-F D$.

We shall denote by

$$
\Phi_{l}(t)=C_{0} \underbrace{\exp (\ldots \exp }_{l \text {-times }}\left(C_{0} t^{\frac{5}{4}}\right) \ldots),
$$


where $C_{0}$ depends on the initial data and its value may vary from line to line up to some absolute constants. We will also make an intensive use of the following trivial facts

$$
\int_{0}^{t} \Phi_{l}(\tau) d \tau \leqslant \Phi_{l}(t) \quad \text { and } \quad \exp \left(\int_{0}^{t} \Phi_{l}(\tau) d \tau\right) \leqslant \Phi_{l+1}(t) .
$$

\section{Littlewood-Paley theory}

Let us recall briefly the classical dyadic partition of the unity, for a proof see for instance [4]: there exists two positive radial functions $\chi \in \mathcal{D}\left(\mathbb{R}^{3}\right)$ and $\varphi \in \mathcal{D}\left(\mathbb{R}^{3} \backslash\{0\}\right)$ such that

$$
\begin{gathered}
\forall \xi \in \mathbb{R}^{3}, \quad \chi(\xi)+\sum_{q \geqslant 0} \varphi\left(2^{-q} \xi\right)=1, \\
\forall \xi \in \mathbb{R}^{3} \backslash\{0\}, \quad \sum_{q \in \mathbb{Z}} \varphi\left(2^{-q} \xi\right)=1, \\
|j-q| \geqslant 2 \Rightarrow \operatorname{Supp} \varphi\left(2^{-j} .\right) \cap \operatorname{Supp} \varphi\left(2^{-q} .\right)=\varnothing, \\
q \geqslant 1 \Rightarrow \operatorname{Supp} \chi \cap \operatorname{Supp} \varphi\left(2^{-q} .\right)=\varnothing .
\end{gathered}
$$

For every $v \in \mathcal{S}^{\prime}\left(\mathbb{R}^{3}\right)$ one defines the non-homogeneous Littlewood-Paley operators by,

$$
\begin{aligned}
\Delta_{q} v & =\varphi\left(2^{-q} \mathrm{D}\right) v=2^{3 q} h\left(2^{q} .\right) * v \quad \text { for } \quad q \geqslant 0, \\
S_{q} v & =\chi\left(2^{-q} \mathrm{D}\right) v=\sum_{-1 \leqslant p \leqslant q-1} \Delta_{p} v=2^{3 q} g\left(2^{q} .\right) * v, \\
\Delta_{-1} v & =S_{0} v, \quad \Delta_{q} v=0 \quad \text { for } \quad q \leqslant-2 .
\end{aligned}
$$

with $h=\mathcal{F}^{-1} \varphi$ and $g=\mathcal{F}^{-1} \chi$. Similarly, we define the homogeneous operators by

$$
\forall q \in \mathbb{Z} \quad \dot{\Delta}_{q} v=\varphi\left(2^{-q} \mathrm{D}\right) v \quad \text { and } \quad \dot{S}_{q} v=\sum_{-\infty \leqslant j \leqslant q-1} \dot{\Delta}_{j} v .
$$

One can easily check that for every tempered distribution $v$, we have

$$
v=\sum_{q \geqslant-1} \Delta_{q} v
$$

and for all $v \in \mathcal{S}^{\prime}\left(\mathbb{R}^{3}\right) /\left\{\mathcal{P}\left[\mathbb{R}^{3}\right]\right\}$

$$
v=\sum_{q \in \mathbb{Z}} \dot{\Delta}_{q} v
$$

where $\mathcal{P}\left[\mathbb{R}^{3}\right]$ is the space of polynomials, see [36]. Furthermore, the Littlewood-Paley decomposition satisfies the property of almost orthogonality for any $u, v \in \mathcal{S}^{\prime}\left(\mathbb{R}^{3}\right)$,

$$
\begin{aligned}
\Delta_{p} \Delta_{j} u=0 & \text { if } & & |p-j| \geqslant 2, \\
\Delta_{p}\left(S_{j-1} u \Delta_{j} v\right)=0 & \text { if } & & |p-j| \geqslant 5 .
\end{aligned}
$$

The following lemma describes how the derivatives act on spectrally localized functions, see for instance [14.

Lemma 2.1 (Bernstein inequalities). There exists a constant $C>0$ such that for all $q, k \in \mathbb{N}, 1 \leqslant$ $a \leqslant b \leqslant \infty$ and for every tempered distribution $u$ we have

$$
\begin{gathered}
\sup _{|\alpha| \leqslant k}\left\|\partial^{\alpha} S_{q} u\right\|_{L^{b}} \leqslant C^{k} 2^{q\left(k+3\left(\frac{1}{a}-\frac{1}{b}\right)\right)}\left\|S_{q} u\right\|_{L^{a}}, \\
C^{-k} 2^{q k}\left\|\dot{\Delta}_{q} u\right\|_{L^{b}} \leqslant \sup _{|\alpha|=k}\left\|\partial^{\alpha} \dot{\Delta}_{q} u\right\|_{L^{b}} \leqslant C^{k} 2^{q k}\left\|\dot{\Delta}_{q} u\right\|_{L^{b}} .
\end{gathered}
$$




\section{Besov spaces}

Based on Littlewood-Paley operators, we can define Besov spaces as follows. Let $(p, r) \in[1,+\infty]^{2}$ and $s \in \mathbb{R}$. The non-homogeneous Besov space $B_{p, r}^{s}$ is the set of tempered distributions $v$ such that

$$
\|v\|_{B_{p, r}^{s}} \triangleq\left\|\left(2^{q s}\left\|\Delta_{q} v\right\|_{L^{p}}\right)_{q \in \mathbb{Z}}\right\|_{\ell^{r}(\mathbb{Z})}<+\infty .
$$

The homogeneous Besov space $\dot{B}_{p, r}^{s}$ is defined as the set of $\mathcal{S}^{\prime}\left(\mathbb{R}^{3}\right) /\left\{\mathcal{P}\left[\mathbb{R}^{3}\right]\right\}$ such that

$$
\|v\|_{\dot{B}_{p, r}^{s}} \triangleq\left\|\left(2^{q s}\left\|\dot{\Delta}_{q} v\right\|_{L^{p}}\right)_{q \in \mathbb{Z}}\right\|_{\ell^{r}(\mathbb{Z})}<+\infty .
$$

We point out that, for all $s \in \mathbb{R}$ the Besov space $B_{2,2}^{s}$ coincides with the Sobolev space $H^{s}$. The following embeddings are an easy consequence of Bernstein inequalities,

$$
B_{p_{1}, r_{1}}^{s} \hookrightarrow B_{p_{2}, r_{2}}^{s+3\left(\frac{1}{p_{2}}-\frac{1}{p_{1}}\right)} \quad p_{1} \leqslant p_{2} \quad \text { and } \quad r_{1} \leqslant r_{2} .
$$

In order to obtain a better description of the regularizing effect of the transport-diffusion equation, we need to use Chemin-Lerner type spaces $\widetilde{L}_{T}^{\rho} B_{p, r}^{s}$, see for instance [4]. Let $T>0, \rho \geqslant 1,(p, r) \in[1, \infty]^{2}$ and $s \in \mathbb{R}$, we denote by $L_{T}^{\rho} B_{p, r}^{s}$ the space of distribution $f$ such that

$$
\|f\|_{L_{T}^{\rho} B_{p, r}^{s}}:=\left\|\left(2^{j s}\left\|\Delta_{j} f\right\|_{L^{p}}\right)_{\ell^{r}}\right\|_{L_{T}^{\rho}}<+\infty .
$$

We say that $f$ belongs to the Chemin-Lerner space $\widetilde{L}_{T}^{\rho} B_{p, r}^{s}$ if

$$
\|f\|_{\widetilde{L}_{T}^{\rho} B_{p, r}^{s}}:=\left\|2^{j s}\right\| \Delta_{j} f\left\|_{L_{T}^{\rho} L^{p}}\right\|_{\ell^{r}}<+\infty .
$$

The relation between these spaces are detailed in the following lemma, which is a direct consequence of the Minkowski inequality.

Lemma 2.2. Let $s \in \mathbb{R}, \varepsilon>0$ and $(p, r, \rho) \in[1,+\infty]^{3}$. Then we have the following embeddings

$$
\begin{gathered}
L_{T}^{\rho} B_{p, r}^{s} \hookrightarrow \widetilde{L}_{T}^{\rho} B_{p, r}^{s} \hookrightarrow L_{T}^{\rho} B_{p, r}^{s-\varepsilon} \quad \text { if } \quad r \geqslant \rho . \\
L_{T}^{\rho} B_{p, r}^{s+\varepsilon} \hookrightarrow \widetilde{L}_{T}^{\rho} B_{p, r}^{s} \hookrightarrow L_{T}^{\rho} B_{p, r}^{s} \quad \text { if } \quad \rho \geqslant r .
\end{gathered}
$$

Now, we introduce the Bony's decomposition [7] which is the basic tool of the para-differential calculus. Formally the product of two tempered distributions $u$ and $v$ can be divided into three parts as follows:

$$
u v=T_{u} v+T_{v} u+R(u, v)
$$

where

$$
T_{u} v=\sum_{q} S_{q-1} u \Delta_{q} v \quad \text { and } \quad R(u, v)=\sum_{q} \Delta_{q} u \widetilde{\Delta}_{q} v, \quad \text { with } \quad \tilde{\Delta}_{q}=\sum_{i=-1}^{1} \Delta_{q+i} .
$$

\section{Some commutator estimates}

The following proposition is proved in [26].

Proposition 2.1. Let $u$ be a smooth function and $v$ be a smooth divergence-free vector field of $\mathbb{R}^{3}$ such that it's vorticity $\omega:=\operatorname{curl} v$ belongs to $L^{\infty}$. Then for every $p \in[1, \infty]$ and $q \geqslant-1$, we have

$$
\left\|\left[\Delta_{q}, v \cdot \nabla\right] u\right\|_{L^{p}} \lesssim\|u\|_{L^{p}}\left(\left\|\nabla \Delta_{-1} v\right\|_{L^{\infty}}+(q+2)\|\omega\|_{L^{\infty}}\right) .
$$


The following proposition is proved in [27]

Proposition 2.2. Let $v$ be an axisymmetric smooth and divergence-free vector field without swirl and let $u$ be a smooth scalar function. Then there exists $C>0$ such that for every $q \in \mathbb{N} \cup\{-1\}$, we have

$$
\left\|\left[\Delta_{q}, v \cdot \nabla\right] u\right\|_{L^{2}} \leqslant C\left\|\frac{\omega^{\theta}}{r}\right\|_{L^{3,1}}\left(\left\|x_{h} u\right\|_{L^{6}}+\|u\|_{L^{2}}\right) .
$$

where $\omega^{\theta}$ is the angular component of $\omega=\nabla \times v$.

The following commutator estimate is proved in [14],

Lemma 2.3. Let $\eta$ be a smooth function and $v$ be a smooth vector field of $\mathbb{R}^{3}$ with zero divergence. Then we have, for all $1 \leqslant p \leqslant \infty$ and $-1<s<1$,

$$
\sum_{j \geqslant-1} 2^{j s}\left\|\left[\Delta_{j}, v \cdot \nabla\right] u\right\|_{L^{p}} \lesssim\|\nabla v\|_{L^{\infty}}\|u\|_{B_{p, 1}^{s}} .
$$

\section{Lorentz spaces and interpolation}

Let $p \in] 1, \infty\left[, q \in[1, \infty]\right.$, The Lorentz space $L^{p, q}$ can be defined by real interpolation from Lebesgue spaces:

$$
\left(L^{p_{0}}, L^{p_{1}}\right)_{(\theta, q)}=L^{p, q}
$$

where $1 \leqslant p_{0}<p<p_{1} \leqslant \infty$ and $\theta$ satisfies $\frac{1}{p}=\frac{1-\theta}{p_{0}}+\frac{\theta}{p_{1}}$ with $0<\theta<1$. The spaces $L^{p, q}$ have the following properties: (see pages 18-20 of [33])

$$
\begin{aligned}
& L^{p, p}=L^{p}, \\
& L^{p, q} \hookrightarrow L^{p, q^{\prime}}, \quad 1 \leqslant q \leqslant q^{\prime} \leqslant \infty, \\
& \|u v\|_{L^{p, q}} \leqslant\|u\|_{L^{\infty}}\|v\|_{L^{p, q}} .
\end{aligned}
$$

Furthermore, for all $1 \leqslant p<3$ we have the embedding, see page 21 of [3],

$$
B_{p, 1}^{\frac{3}{p}-1} \hookrightarrow L^{3,1} .
$$

\section{Estimates for a transport equation}

We need the following proposition which deals with the persistence of Besov regularities in a transport equation. Abidi, Hmidi and Keraani, in [3], proved the result for the case $s= \pm 1$. The remainder cases are done in [14, 44].

Proposition 2.3 (Proposition A.2 in [3]). Let $s \in(-1,1), p, r \in[1, \infty]$ and $v$ be a smooth divergencefree vector field. Let $f$ be a smooth solution of the equation

$$
\partial_{t} f+v \cdot \nabla f=g, \quad f_{\mid t=0}=f_{0},
$$

where $f^{0} \in B_{p, r}^{s}\left(\mathbb{R}^{3}\right)$ and $g \in L_{\text {loc }}^{1}\left(\mathbb{R}_{+}, B_{p, r}^{s}\right)$. Then for all $t \in \mathbb{R}_{+}$, we have

$$
\|f(t)\|_{B_{p, r}^{s}} \lesssim e^{C U(t)}\left(\left\|f^{0}\right\|_{B_{p, r}^{s}}+\int_{0}^{t} e^{-C U(\tau)}\|g(\tau)\|_{B_{p, r}^{s}} d \tau\right),
$$

with $U(t):=\|\nabla v(t)\|_{L_{t}^{1} L^{\infty}}$ and $C$ is a constant depending on $s$. The above estimate is true in the two following case

$$
s=-1, r=\infty, 1 \leqslant p \leqslant \infty \quad \text { and } \quad s=r=1,1 \leqslant p \leqslant \infty,
$$

provided that we change $U(t)$ by $V(t):=\|v(t)\|_{L_{t}^{1} B_{\infty, 1}^{1}}$. 
We recall the following Nash-De Giorgi estimate for the convection-diffussion equation.

Proposition 2.4 (Lemma A.1 in [27]). Consider the equation

$$
\partial_{t} f+v \cdot \nabla f-\Delta f=\nabla \cdot F+G, \quad t>0, \quad x \in \mathbb{R}^{3}, \quad f_{\mid t=0}=f_{0} .
$$

Consider $p, q, p_{1}, q_{1} \in[0, \infty], r \in[2, \infty]$ with $\frac{2}{p}+\frac{3}{q}<1, \frac{2}{p_{1}}+\frac{3}{q_{1}}<2$. There exists $C>0$ such that for every smooth divergence-free vector field $v$ and every $T>0$, if $F \in L_{T}^{p} L^{q}, G \in L_{T}^{p_{1}} L^{q_{1}}$ and $f_{0} \in L^{r}$ then the solution of (2.5) satisfies the estimate:

$$
\|f(T)\|_{L^{\infty}} \leqslant C\left(1+T^{-\frac{3}{2 r}}\right)\left\|f_{0}\right\|_{L^{r}}+C\left(1+\sqrt{T}^{1-\left(\frac{2}{p}+\frac{3}{q}\right)}\right)\|F\|_{L_{T}^{p} L^{q}}+C\left(1+\sqrt{T}^{2-\left(\frac{2}{p_{1}}+\frac{3}{q_{1}}\right)}\right)\|G\|_{L_{T}^{p_{1}} L^{q_{1}}} .
$$

\section{Axisymmetric flows}

Along this paper, we assume that vector fields $v: \mathbb{R}^{3} \rightarrow \mathbb{R}^{3}$ and $b: \mathbb{R}^{3} \rightarrow \mathbb{R}^{3}$ are axisymmetric, namely,

$$
\mathcal{R}_{-\alpha}\left\{v\left(\mathcal{R}_{\alpha} x\right)\right\}=v(x), \quad \text { and } \quad \mathcal{R}_{-\alpha}\left\{b\left(\mathcal{R}_{\alpha} x\right)\right\}=b(x), \quad \forall \alpha \in[0,2 \pi], \quad \forall x \in \mathbb{R}^{3},
$$

where $\mathcal{R}_{\alpha}$ denotes the rotation around the axis $(O z)$ with angle $\alpha$. The vector field $v$ is assumed to be without swirl and the radial and the height components of magnetic velocity field $b$ are trivial, that is,

$$
v(x)=v^{r}(r, z) e_{r}+v^{z}(r, z) e_{z}, \quad b(x)=b^{\theta}(r, z) e_{\theta},
$$

where $x=\left(x_{1}, x_{2}, z\right), r=\left(x_{1}^{2}+x_{2}^{2}\right)^{\frac{1}{2}}$, and $\left(e_{r}, e_{\theta}, e_{z}\right)$ is the cylindrical basis of $\mathbb{R}^{3}$,

$$
e_{r}:=(\cos \theta, \sin \theta, 0)^{T}, \quad e_{\theta}:=(-\sin \theta, \cos \theta, 0)^{T}, \quad e_{z}:=(0,0,1)^{T} .
$$

The components $v^{r}, v^{z}$ and $b^{\theta}$ do not depend on the angular variable $\theta$. Recall that, in the cylindrical coordinate systems, the gradient operator $\nabla$ is given by

$$
\nabla=e_{r} \partial_{r}+\frac{1}{r} e_{\theta} \partial_{\theta}+e_{z} \partial_{z}
$$

and the expression of the Laplacian operator is given by

$$
\Delta=\partial_{r r}+\frac{1}{r} \partial_{r}+\partial_{z z}
$$

The incompressible constraints, $\operatorname{div} v=0$ and $\operatorname{div} b=0$, can be written as

$$
\partial_{r} v^{r}+\frac{1}{r} v^{r}+\partial_{z} v^{z}=0, \quad \text { and } \quad \partial_{r} b^{r}+\frac{1}{r} b^{r}+\partial_{z} b^{z}=0 .
$$

Moreover, for any axisymmetric vector filed $a$, one has

$$
a \cdot \nabla=a^{r} \partial_{r}+\frac{a^{\theta}}{r} \partial_{\theta}+a^{z} \partial_{z} .
$$

Thus, straightforward computations show that the equations (1.1) can be expressed in cylindrical coordinate as

$$
\left\{\begin{array}{l}
\partial_{t} v^{r}+v^{r} \partial_{r} v^{r}+v^{z} \partial_{z} v^{r}+\partial_{r} p=-\frac{\left(b^{\theta}\right)^{2}}{r} \\
\partial_{t} v^{z}+v^{r} \partial_{r} v^{z}+v^{z} \partial_{z} v^{z}+\partial_{z} p=0 \\
\partial_{t} b^{\theta}+v^{r} \partial_{r} b^{\theta}+v^{z} \partial_{z} b^{\theta}-\Delta b^{\theta}=\frac{b^{\theta}}{r} v^{r} .
\end{array}\right.
$$


Furthermore, one can easily check that the vorticity $\omega:=\operatorname{curl} v$ of the vector filed $v$ is given by

$$
\omega=\omega^{\theta} e_{\theta} ; \quad \omega^{\theta}=\partial_{z} v^{r}-\partial_{r} v^{z}
$$

Now, applying the curl operator to the velocity equation yields

$$
\partial_{t} \omega+v \cdot \nabla \omega=\omega \cdot \nabla v+\operatorname{curl}(b \cdot \nabla b) .
$$

Since $\omega=\omega^{\theta} e_{\theta}$ and by (3.5), the stretching term takes the form

$$
\omega \cdot \nabla v=\omega^{\theta} \frac{v^{r}}{r} e_{\theta}=\frac{v^{r}}{r} \omega
$$

Similarly, as $b=b^{\theta} e_{\theta}$ we get

$$
b \cdot \nabla b=-\frac{\left(b^{\theta}\right)^{2}}{r} e_{r} \quad \text { and } \quad \operatorname{curl}(b \cdot \nabla b)=-\partial_{z}\left(\frac{b^{\theta}}{r} b\right) .
$$

Consequently the vorticity equation (3.7) becomes

$$
\partial_{t} \omega+v \cdot \nabla \omega=\frac{v^{r}}{r} \omega-\partial_{z}\left(\frac{b^{\theta}}{r} b\right) .
$$

It follows that the scalar function $\omega^{\theta}$ verifies

$$
\partial_{t} \omega^{\theta}+v \cdot \nabla \omega^{\theta}=\frac{v^{r}}{r} \omega^{\theta}-\partial_{z} \frac{\left(b^{\theta}\right)^{2}}{r} .
$$

Moreover, from the third equation of (3.6), one has

$$
\partial_{t} b^{\theta}+v \cdot \nabla b^{\theta}-\Delta b^{\theta}=\frac{b^{\theta}}{r} v^{r} .
$$

Therefore the quantities $\Omega:=\frac{\omega^{\theta}}{r}$ and $\Gamma:=\frac{b^{\theta}}{r}$ are governed by the following system

$$
\left\{\begin{array}{l}
\partial_{t} \Omega+v \cdot \nabla \Omega=-\partial_{z} \Gamma^{2} \\
\partial_{t} \Gamma+v \cdot \nabla \Gamma-\left(\Delta+\frac{2}{r} \partial_{r}\right) \Gamma=0 .
\end{array}\right.
$$

The following proposition is proved in [3, 24].

Proposition 3.1. Let $v=\left(v^{1}, v^{2}, v^{3}\right)$ and $b=\left(b^{1}, b^{2}, b^{3}\right)$ be a smooth axisymmetric vector fields as in (3.1). Then we have

(i) For every $\left(x_{1}, x_{2}, z\right) \in \mathbb{R}^{3}$,

$$
\begin{aligned}
v^{z} & =0, \quad-x_{2} v^{1}\left(x_{1}, x_{2}, z\right)+x_{1} v^{2}\left(x_{1}, x_{2}, z\right)=0 \\
b^{z} & =0, \quad x_{1} b^{1}\left(x_{1}, x_{2}, z\right)+x_{2} b^{2}\left(x_{1}, x_{2}, z\right)=0 \\
v^{1}\left(0, x_{2}, z\right) & =v^{2}\left(x_{1}, 0, z\right)=b^{1}\left(x_{1}, 0, z\right)=b^{2}\left(0, x_{2}, z\right)=0 .
\end{aligned}
$$

(ii) For every $q \geqslant-1, \Delta_{q} v$ and $\Delta_{q} b$ are axisymmetric and

$$
\begin{aligned}
& \left(\Delta_{q} v^{1}\right)\left(0, x_{2}, z\right)=\left(\Delta_{q} v^{2}\right)\left(x_{1}, 0, z\right)=0, \\
& \left(\Delta_{q} b^{1}\right)\left(x_{1}, 0, z\right)=\left(\Delta_{q} b^{2}\right)\left(0, x_{2}, z\right)=0 .
\end{aligned}
$$


(iii) The vector $\omega=\nabla \times v=\left(\omega^{1}, \omega^{2}, \omega^{3}\right)$ satisfies $\omega=\nabla \times e_{\theta}=(0,0,0)$ and we have for every $\left(x_{1}, x_{2}, z\right) \in \mathbb{R}^{3}$,

$$
\omega^{z}=0, \quad x_{1} \omega^{1}\left(x_{1}, x_{2}, z\right)+x_{2} \omega^{2}\left(x_{1}, x_{2}, z\right)=0, \quad \omega^{1}\left(x_{1}, 0, z\right)=\omega^{2}\left(0, x_{2}, z\right)=0 .
$$

Now, we aim to study the following vorticity like equation, in which no relations between the vector field $v$ and the solution $\zeta$ are supposed

$$
\left\{\begin{array}{l}
\partial_{t} \zeta+(v \cdot \nabla) \zeta=(\zeta \cdot \nabla) v+\operatorname{curl}(b \cdot \nabla b), \\
\operatorname{div} v=0 \\
\zeta_{\mid t=0}=\zeta_{0}
\end{array}\right.
$$

where $v$ and $b$ are axisymmetric and verify (3.1) and $\zeta=\left(\zeta^{1}, \zeta^{2}, \zeta^{z}\right)$ is an unknown vector field.

Proposition 3.2. Let $v$ be a smooth, divergence free, axisymmetric vector field and $\zeta$ be the unique global solution of (3.14) with smooth initial data $\zeta_{0}$. Then we have the following properties.

(i) If $\operatorname{div} \zeta_{0}=0$, then for all $t \in \mathbb{R}_{+}$, $\operatorname{div} \zeta(t)=0$.

(ii) If $\zeta_{0} \times \vec{e}_{\theta}=0$ then for all $t \in \mathbb{R}_{+}, \zeta(t, x) \times \vec{e}_{\theta}=(0,0,0)$. Consequently,

$$
\zeta_{1}\left(t, x_{1}, 0, z\right)=\zeta_{2}\left(t, 0, x_{2}, z\right)=0 \quad \text { and } \quad(\zeta \cdot \nabla) v=\frac{v^{r}}{r} \zeta .
$$

Proof. We apply the divergence operator to the equation (3.14), we get

$$
\partial_{t}(\operatorname{div} \zeta)+\operatorname{div}(v \cdot \nabla \zeta)=\operatorname{div}(\zeta \cdot \nabla v)
$$

where we have used the fact that $\operatorname{div}(\operatorname{curl} a)=0$ for any vector $a \in \mathbb{R}^{3}$. Straightforward computations allows to get

$$
\operatorname{div}(v \cdot \nabla \zeta)-\operatorname{div}(\zeta \cdot \nabla v)=v \cdot \nabla \operatorname{div} \zeta
$$

Thus we obtain the equation

$$
\partial_{t} \operatorname{div} \zeta+(v \cdot \nabla) \operatorname{div} \zeta=0 .
$$

Then, by the maximum principle we conclude (i).

Next, we shall prove (ii). Denote by $\left(\zeta^{r}, \zeta^{\theta}, \zeta^{z}\right)$ the coordinates of $\zeta$ in cylindrical basis. Then

$$
\zeta \times \vec{e}_{\theta}=\zeta^{r} e_{z}-\zeta^{z} e_{r}
$$

Hence it suffices to prove that $\zeta^{r}=\zeta^{z}=0$. Taking the scalar product of (3.14) with $e_{r}$ we get

$$
\partial_{t} \zeta^{r}+(v \cdot \nabla \zeta) \cdot e_{r}=(\zeta \cdot \nabla v) \cdot e_{r}+\operatorname{curl}(b \cdot \nabla b) \cdot e_{r}
$$

From (3.5) and since $\partial_{r} e_{r}=\partial_{z} e_{r}=0$, we get

$$
\begin{aligned}
(v \cdot \nabla \zeta) \cdot e_{r} & =\left(v^{r} \partial_{r} \zeta+v^{3} \partial_{z} \zeta\right) \cdot e_{r} \\
& =\left(v^{r} \partial_{r}+v^{3} \partial_{z}\right)\left(\zeta \cdot e_{r}\right) \\
& =v \cdot \nabla \zeta^{r}
\end{aligned}
$$

Similarly we obtain

$$
(\zeta \cdot \nabla v) \cdot e_{r}=\left(\zeta^{r} \partial_{r} v+\frac{1}{r} \zeta^{\theta} \partial_{z} v+\zeta^{z} \partial_{3} v\right) \cdot e_{r}=\zeta^{r} \partial_{r}+\zeta^{z} \partial_{z} v^{r}
$$


On the other hand, by (3.1) and (3.9) we have

$$
\operatorname{curl}(b \cdot \nabla b) \cdot e_{r}=0 .
$$

Thus $\zeta^{r}$ verifies the equation

$$
\partial_{t} \zeta^{r}+v \cdot \nabla \zeta^{r}=\zeta^{r} \partial_{r} v^{r}+\zeta^{z} \partial_{z} v^{r}
$$

By the maximum principle we get

$$
\left\|\zeta^{r}(t)\right\|_{L^{\infty}} \leqslant \int_{0}^{t}\left(\left\|\zeta^{r}(\tau)\right\|_{L^{\infty}}+\left\|\zeta^{z}(\tau)\right\|_{L^{\infty}}\right)\|\nabla v(\tau)\|_{L^{\infty}} d \tau .
$$

In a similar way we may check that

$$
\partial_{t} \zeta^{z}+v \cdot \nabla \zeta^{z}=\zeta^{r} \partial_{r} v^{3}+\zeta^{z} \partial_{z} v^{3} .
$$

Therefore, we have

$$
\left\|\zeta^{z}(t)\right\|_{L^{\infty}} \leqslant \int_{0}^{t}\left(\left\|\zeta^{r}(\tau)\right\|_{L^{\infty}}+\left\|\zeta^{z}(\tau)\right\|_{L^{\infty}}\right)\|\nabla v(\tau)\|_{L^{\infty}} d \tau .
$$

Combining the two last estimates with Gronwall's inequality, we obtain for every $t \in \mathbb{R}+$,

$$
\zeta^{r}(t)=\zeta^{z}(t)=0
$$

It follows that $\zeta \times e_{\theta}=0$ and the the stretching term becomes

$$
\zeta \cdot \nabla v=\zeta^{\theta} \frac{v^{r}}{r} e_{\theta}=\frac{v^{r}}{r} \zeta .
$$

The proof of the Proposition is now complete.

\section{A priori estimates}

In this section we shall establish some global a priori estimates needed for the proof of Theorem 1.1 and Theorem 1.2. First, we shall prove some basic weak estimates that can be easily obtained through energy type estimates. In a second step, we shall give a global estimate on $\|\omega / r\|_{L_{t}^{\infty} L^{3,1}}$ and finally we intend to show the control of some stronger norms such as $\|\omega\|_{L_{t}^{\infty} L^{\infty}}$ and $\|b\|_{L_{t}^{1} B_{\infty, 1}^{0}}$.

Recall that we always denote

$$
\Phi_{l}(t)=C_{0} \underbrace{\exp (\ldots \exp }_{l-\text { times }}\left(C_{0} t^{\frac{5}{4}}\right) \ldots),
$$

where $C_{0}$ depends on the involved norms of the initial data and its value may vary from line to line up to some absolute constants.

We start with the following elementary estimates.

Proposition 4.1. Let $(v, b)$ be a smooth solution of (1.1) then

(i) For $v_{0}, b_{0} \in L^{2}$ and $t \in \mathbb{R}_{+}$we have the energy law of the three-dimensional incompressible $M H D$ equations

$$
\|v\|_{L_{t}^{\infty} L^{2}}^{2}+\|b\|_{L_{t}^{\infty} L^{2}}^{2}+2\|\nabla b\|_{L_{t}^{2} L^{2}}^{2}=\left\|v_{0}\right\|_{L^{2}}^{2}+\left\|b_{0}\right\|_{L^{2}}^{2} .
$$


(ii) Assume that $(v, b)$ is an axisymmetric solution of (1.1). Then, for all $t \in \mathbb{R}_{+}$we have

$$
\left\|\frac{b^{\theta}}{r}\right\|_{L_{t}^{\infty} L^{2}}^{2}+\left\|\nabla\left(\frac{b^{\theta}}{r}\right)\right\|_{L_{t}^{2} L^{2}}^{2} \leqslant\left\|\frac{b_{0}^{\theta}}{r}\right\|_{L^{2}}^{2} .
$$

In addition, for all $p \in(1, \infty], q \in[1, \infty]$ and $t \in \mathbb{R}_{+}$,

$$
\left\|\frac{b^{\theta}}{r}\right\|_{L_{t}^{\infty} L^{p, q}} \leqslant\left\|\frac{b_{0}^{\theta}}{r}\right\|_{L^{p, q}} .
$$

(iii) Assume that $(v, b)$ is an axisymmetric solution of (1.1) such that $v_{0}, b_{0} \in L^{2}$, and $b_{0}^{\theta} / r \in L^{m}$, $m>6$. Then for all $t \in \mathbb{R}_{+}$we have

$$
\|b(t)\|_{L^{\infty}} \leqslant C_{0}\left(t^{-\frac{3}{4}}+t^{\frac{1}{4}}\right),
$$

and

$$
\|b\|_{L_{t}^{1} L^{p}} \leqslant C_{0}\left(t^{\frac{1}{4}}+t^{\frac{5}{4}}\right) \text { for all } p \in(2, \infty] .
$$

Proof. By taking the $L^{2}$-scalar product of the first equation of (1.1) with $v$ and integrating by parts we get, since $v$ and $b$ are divergence free,

$$
\frac{1}{2} \frac{d}{d t}\|v(t)\|_{L^{2}}^{2}=-\int_{\mathbb{R}^{3}}(b \cdot \nabla v) \cdot b d x .
$$

Making a similar energy estimate on the second equation of (1.1) yields

$$
\frac{1}{2} \frac{d}{d t}\|b(t)\|_{L^{2}}^{2}+\int_{\mathbb{R}^{3}}|\nabla b(t, x)|^{2} d x=\int_{\mathbb{R}^{3}}(b \cdot \nabla v) \cdot b d x .
$$

Summing the two last identities gives

$$
\frac{1}{2} \frac{d}{d t}\|v(t)\|_{L^{2}}^{2}+\frac{1}{2} \frac{d}{d t}\|b(t)\|_{L^{2}}^{2}+\int_{\mathbb{R}^{3}}|\nabla b(t, x)|^{2} d x=0 .
$$

Integrating in time gives the desired result in (i).

Recall that the evolution of the quantity $\Gamma:=b^{\theta} / r$ is given by

$$
\partial_{t} \Gamma+v \cdot \nabla \Gamma-\left(\Delta+\frac{2}{r} \partial_{r}\right) \Gamma=0 .
$$

Multiplying the last equation by $\Gamma$ an integrating in space we get

$$
\frac{1}{2} \frac{d}{d t}\|\Gamma(t)\|_{L^{2}}^{2}+\|\nabla \Gamma(t)\|_{L^{2}}^{2}+2 \pi \int_{\mathbb{R}}|\Gamma(t, 0, z)| d z=0 .
$$

Similarly, for all $p \in[1, \infty]$ we have

$$
\|\Gamma(t)\|_{L^{p}} \leqslant\left\|\Gamma_{0}\right\|_{L^{p}}
$$

By interpolation, for $p \in] 1, \infty[$ and $q \in[1, \infty]$, we conclude (ii).

Now we shall prove (iii). Since $b=b^{\theta} e_{\theta}$ then from (3.12) we have

$$
\partial_{t} b+v \cdot \nabla b-\Delta b=\frac{b}{r} v^{r} .
$$


Applying Proposition 2.4 to (4.7) with $F=0$ and $G=\frac{b}{r} v_{r}$ we get, for all $m>6$,

$$
\begin{aligned}
\|b(t)\|_{L^{\infty}} & \leqslant C\left(1+t^{-\frac{3}{4}}\right)\left\|b_{0}\right\|_{L^{2}}+C\left(1+t^{\left(\frac{1}{4}-\frac{3}{2 m}\right)}\right)\left\|v^{r} \frac{b}{r}\right\|_{L_{t}^{\infty} L^{\frac{2 m}{m+2}}} \\
& \leqslant C\left(1+t^{-\frac{3}{4}}\right)\left\|b_{0}\right\|_{L^{2}}+C\left(1+t^{\left(\frac{1}{4}-\frac{3}{2 m}\right)}\right)\|v\|_{L_{t}^{\infty} L^{2}}\left\|\frac{b^{\theta}}{r}\right\|_{L_{t}^{\infty} L^{m}} .
\end{aligned}
$$

Then, by (4.1) and (4.3) we conclude that

$$
\|b(t)\|_{L^{\infty}} \leqslant C\left(\left\|b_{0}\right\|_{L^{2}}+\left\|v_{0}\right\|_{L^{2}}\right)\left(1+\left\|\frac{b_{0}^{\theta}}{r}\right\|_{L^{m}}\right)\left(t^{-\frac{3}{4}}+t^{\frac{1}{4}}\right) .
$$

The estimate (4.5) can be immediately obtained by using an interpolation inequality in the $L^{p}$ spaces, with $p \in(2, \infty)$, the dispersive estimate (4.8), the energy estimate (4.1) and finally integration in time.

The following Proposition provides the main important quantities that one should estimate in order to get the global existence of smooth solutions.

Proposition 4.2. Let $v_{0}, b_{0} \in L^{2}$ be two smooth axisymmetric vector fields with zero divergence such that $\omega_{0}^{\theta} / r \in L^{3,1}$ and $b_{0}^{\theta} / r \in L^{2} \cap L^{\infty}$. Then we have for every $t \in \mathbb{R}_{+}$,

$$
\left\|\frac{v^{r}}{r}\right\|_{L_{t}^{\infty} L^{\infty}}+\left\|\frac{\omega}{r}\right\|_{L_{t}^{\infty} L^{3,1}}+\|\Gamma\|_{L_{t}^{1} B_{2,1}^{\frac{3}{2}}} \leqslant \Phi_{1}(t) .
$$

Proof. We use the following inequality due to Shirota and Yanagisawa [42]. See also [3].

$$
\left|\frac{v^{r}}{r}\right| \lesssim \frac{1}{|.|^{2}} *\left|\frac{\omega^{\theta}}{r}\right|
$$

Since $\frac{1}{|.|^{2}} \in L^{\frac{3}{2}, \infty}$ then by the convolution law $L^{p, q} * L^{p^{\prime}, q^{\prime}} \rightarrow L^{\infty}$ we get

$$
\left\|\frac{v^{r}}{r}\right\|_{L^{\infty}} \lesssim\left\|\frac{\omega^{\theta}}{r}\right\|_{L^{3,1}}
$$

On the other hand, we recall that the evolution of the quantity $\Omega:=\omega^{\theta} / r$ is given by

$$
\partial_{t} \Omega+v \cdot \nabla \Omega=-\partial_{z} \Gamma^{2}
$$

with $\Gamma:=b^{\theta} / r$. By making an $L^{p}$ estimates and interpolating we get

$$
\begin{aligned}
\|\Omega(t)\|_{L^{3,1}} & \leqslant\left\|\Omega_{0}\right\|_{L^{3,1}}+\int_{0}^{t}\left\|\partial_{z} \Gamma^{2}(\tau)\right\|_{L^{3,1}} d \tau \\
& \leqslant\left\|\Omega_{0}\right\|_{L^{3,1}}+2 \int_{0}^{t}\|\Gamma(\tau)\|_{L^{\infty}}\left\|\partial_{z} \Gamma(\tau)\right\|_{L^{3,1}} d \tau,
\end{aligned}
$$

where we have used in the last estimate the inequality (2.3). Then using the embedding (2.4) and Proposition 4.17(ii) we find

$$
\|\Omega(t)\|_{L^{3,1}} \leqslant\left\|\Omega_{0}\right\|_{L^{3,1}}+2\left\|\Gamma_{0}\right\|_{L^{\infty}}\|\Gamma\|_{L_{t}^{1} B_{2,1}^{\frac{3}{2}}}
$$


Now, we shall estimate $\|\Gamma\|_{L_{t}^{1} B_{2,1}^{\frac{3}{2}}}$. Let $q \in \mathbb{T}$ and set $\Gamma_{q}:=\Delta_{q} \Gamma$. Then, localizing in frequency the second equation of (3.13) gives

$$
\partial_{t} \Gamma_{q}+v \cdot \nabla \Gamma_{q}-\left(\Delta+\frac{2}{r} \partial_{r}\right) \Gamma_{q}=-\left[\Delta_{q}, v \cdot \nabla\right] \Gamma .
$$

Multiplying (4.11) by $\left|\Gamma_{q}\right|^{p-2} \Gamma_{q}, p \geqslant 2$, and using Hölder inequality, we get

$$
\frac{1}{p} \frac{d}{d t}\left\|\Gamma_{q}(t)\right\|_{L^{p}}^{p}-\int_{\mathbb{R}^{3}}\left(\Delta \Gamma_{q}\right)\left|\Gamma_{q}\right|^{p-2} \Gamma_{q} d x-\int_{\mathbb{R}^{3}} \frac{2}{r} \partial_{r} \Gamma_{q}\left|\Gamma_{q}\right|^{p-2} \Gamma_{q} d x \leqslant\left\|\Gamma_{q}\right\|_{L^{p}}^{p-1}\left\|\left[\Delta_{q}, v \cdot \nabla\right] \Gamma\right\|_{L^{p}} .
$$

Obviously,

$$
0 \leqslant-\int_{\mathbb{R}^{3}} \frac{2}{r} \partial_{r} \Gamma_{q}\left|\Gamma_{q}\right|^{p-2} \Gamma_{q} d x .
$$

Moreover, using the generalized Bernstein inequality, see [33], we find

$$
\frac{1}{p} 2^{2 q}\left\|\Gamma_{q}(t)\right\|_{L^{p}}^{p} \leqslant-\int_{\mathbb{R}^{3}}\left(\Delta \Gamma_{q}\right)\left|\Gamma_{q}\right|^{p-2} \Gamma_{q} d x
$$

Combining the three last estimates gives

$$
\frac{d}{d t}\left\|\Gamma_{q}(t)\right\|_{L^{p}}+c_{p} 2^{2 q}\left\|\Gamma_{q}\right\|_{L^{p}} \lesssim\left\|\left[\Delta_{q}, v \cdot \nabla\right] \Gamma\right\|_{L^{p}}
$$

This leads to

$$
\left\|\Gamma_{q}(t)\right\|_{L^{p}} \leqslant e^{-c_{p} t 2^{2 q}}\left\|\Delta_{q} \Gamma_{0}\right\|_{L^{p}}+\int_{0}^{t} e^{-c_{p} 2^{2 q}(t-\tau)}\left\|\left[\Delta_{q}, v \cdot \nabla\right] \Gamma\right\|_{L^{p}} d \tau .
$$

Therefore, by integration in time, we obtain

$$
\left\|\Gamma_{q}\right\|_{L_{t}^{\infty} L^{p}}+2^{2 q}\left\|\Gamma_{q}\right\|_{L_{t}^{1} L^{p}} \lesssim\left\|\Gamma_{0}\right\|_{L^{p}}+\left\|\left[\Delta_{q}, v \cdot \nabla\right] \Gamma\right\|_{L_{t}^{1} L^{p}}
$$

In particular, for $p=2$, we have

$$
\left\|\Gamma_{q}\right\|_{L_{t}^{1} L^{2}} \lesssim 2^{-2 q}\left\|\Gamma_{0}\right\|_{L^{2}}+2^{-2 q}\left\|\left[\Delta_{q}, v \cdot \nabla\right] \Gamma\right\|_{L_{t}^{1} L^{2}} .
$$

Multiplying this last inequality by $2^{q \frac{3}{2}}$, then summing over $q$ we find

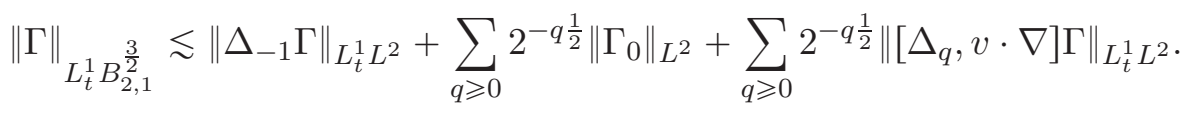

Then, we shall use Proposition 2.2 and Proposition 4.1.(ii) to obtain

$$
\begin{aligned}
\|\Gamma\|_{L_{t}^{1} B_{2,1}^{\frac{3}{2}}} & \lesssim\left\|\Gamma_{0}\right\|_{L^{2}}+\|\Gamma\|_{L_{t}^{1} L^{2}}+\int_{0}^{t}\|\Omega(\tau)\|_{L^{3,1}}\left(\left\|b^{\theta}(\tau)\right\|_{L^{6}}+\|\Gamma(\tau)\|_{L^{2}}\right) d \tau \\
& \lesssim\left\|\Gamma_{0}\right\|_{L^{2}}(t+1)+\int_{0}^{t}\|\Omega(\tau)\|_{L^{3,1}}\left(\left\|b^{\theta}(\tau)\right\|_{L^{6}}+\left\|\Gamma_{0}\right\|_{L^{2}}\right) d \tau
\end{aligned}
$$

Inserting the last estimate into (4.10) gives

$$
\|\Omega(t)\|_{L^{3,1}} \leqslant C_{0}(t+1)+C_{0} \int_{0}^{t}\|\Omega(\tau)\|_{L^{3,1}}\left(\left\|b^{\theta}(\tau)\right\|_{L^{6}}+1\right) d \tau .
$$


The Gronwall lemma and Proposition 4.17(iii) yield that

$$
\|\Omega(t)\|_{L^{3,1}} \leqslant C_{0}(t+1) e^{C_{0}\left\|b^{\theta}\right\|_{L_{t}^{1} L^{6}}} \leqslant C_{0} e^{C_{0} t^{\frac{5}{4}}} .
$$

This gives in turn, by (4.13) and Proposition 4.17(iii),

$$
\|\Gamma\|_{L_{t}^{1} B_{2,1}^{\frac{3}{2}}} \leqslant C_{0}(t+1)+C_{0}\left(1+t^{\frac{5}{4}}\right) e^{C_{0} t^{\frac{5}{4}}} \leqslant \Phi_{1}(t) .
$$

Moreover, by (4.9), we get

$$
\left\|\frac{v^{r}}{r}(t)\right\|_{L^{\infty}} \lesssim\|\Omega(t)\|_{L^{3,1}} \leqslant \Phi_{1}(t) .
$$

Finally, using the fact that $\omega=\omega^{\theta} e_{\theta}$ and from (2.3) we infer that

$$
\left\|\frac{\omega}{r}(t)\right\|_{L^{3,1}} \lesssim\|\Omega(t)\|_{L^{3,1}} \leqslant \Phi_{1}(t) .
$$

This concludes the proof of the proposition.

With the estimates established in Proposition 4.1 and Proposition 4.2 in hand, we can bound $\|\omega\|_{L_{t}^{\infty} L^{\infty}}$ by using the smoothing effect on the magnetic field.

Proposition 4.3. Let $\sigma \in(3, \infty), v_{0} \in L^{2}$ and $b_{0} \in L^{2} \cap B_{\sigma, 1}^{3 / \sigma-1}$ be two smooth axisymmetric vector fields with zero divergence such that $\omega_{0} \in L^{\infty}, \omega_{0}^{\theta} / r \in L^{3,1}$ and $b_{0}^{\theta} / r \in L^{2} \cap L^{\infty}$. Then we have for every $t \in \mathbb{R}+$,

$$
\|b\|_{L_{t}^{\infty} B_{\sigma, 1}^{3 / \sigma-1}}+\|b\|_{L_{t}^{1} B_{\sigma, 1}^{3 / \sigma+1}}+\|\omega\|_{L_{t}^{\infty} L^{\infty}}+\|b\|_{L_{t}^{2} B_{\infty, 1}^{0}} \leqslant \Phi_{2}(t) .
$$

Proof. Applying the maximum principle to 3.10 gives

$$
\begin{aligned}
\|\omega(t)\|_{L^{\infty}} & \lesssim\left\|\omega_{0}\right\|_{L^{\infty}}+\int_{0}^{t}\left\|\partial_{z} b(\tau)\right\|_{L^{\infty}}\left\|b^{\theta}(\tau) / r\right\|_{L^{\infty}} d \tau+\int_{0}^{t}\left\|v^{r}(\tau) / r\right\|_{L^{\infty}}\|\omega(\tau)\|_{L^{\infty}} d \tau \\
& \lesssim\left\|\omega_{0}\right\|_{L^{\infty}}+\left\|b_{0}^{\theta} / r\right\|_{L^{\infty}}\|b\|_{L_{t}^{1} B_{\sigma, 1}^{\frac{3}{\sigma}+1}}+\int_{0}^{t}\left\|v^{r}(\tau) / r\right\|_{L^{\infty}}\|\omega(\tau)\|_{L^{\infty}} d \tau
\end{aligned}
$$

where we have used in the last estimate the embedding $B_{\sigma, 1}^{\frac{3}{\sigma}+1} \hookrightarrow \operatorname{Lip}\left(\mathbb{R}^{3}\right)$ and Proposition 4.1 (ii). Now we shall estimate $\|b\|_{L_{t}^{1} B_{\sigma, 1}^{\frac{3}{\sigma}+1}}$. Localizing in frequency the equation (4.7) gives

$$
\partial_{t} b_{q}+v \cdot \nabla b_{q}-\Delta b_{q}=F_{q}-\left[\Delta_{q}, v \cdot \nabla\right] b
$$

for all $q \in \mathbb{N} \cup\{-1\}$ where $b_{q}:=\Delta_{q} b$ and $F_{q}:=\Delta_{q}(b \cdot \nabla v)=\Delta_{q}\left(\frac{v^{r}}{r} b\right)$. Then taking the standard $L^{p}$-estimate yields, for all $p \geqslant 2$,

$$
\frac{1}{p} \frac{d}{d t}\left\|b_{q}(t)\right\|_{L^{p}}^{p}-\int_{\mathbb{R}^{3}}\left(\Delta b_{q}\right)\left|b_{q}\right|^{p-2} b_{q} d x \leqslant\left\|b_{q}(t)\right\|_{L^{p}}^{p-1}\left(\left\|F_{q}(t)\right\|_{L^{p}}+\left\|\left[\Delta_{q}, v \cdot \nabla\right] b(\tau)\right\|_{L^{p}}\right) .
$$

Using the generalized Bernestein inequality, see [33],

$$
\frac{1}{p} 2^{2 q}\left\|b_{q}(t)\right\|_{L^{p}}^{p} \leqslant-\int_{\mathbb{R}^{3}}\left(\Delta b_{q}\right)\left|b_{q}\right|^{p-2} b_{q} d x
$$


we obtain

$$
\frac{d}{d t}\left\|b_{q}(t)\right\|_{L^{p}}+c_{p} 2^{2 q}\left\|b_{q}(t)\right\|_{L^{p}} \lesssim\left\|F_{q}(t)\right\|_{L^{p}}+\left\|\left[\Delta_{q}, v \cdot \nabla\right] b(t)\right\|_{L^{p}} .
$$

This leads to

$$
\left\|b_{q}(t)\right\|_{L^{p}} \lesssim e^{-c_{p} t 2^{2 q}}\left\|b_{q}(0)\right\|_{L^{p}}+\int_{0}^{t} e^{-c(t-\tau) 2^{2 q}}\left(\left\|F_{q}(\tau)\right\|_{L^{p}}+\left\|\left[\Delta_{q}, v \cdot \nabla\right] b(\tau)\right\|_{L^{p}}\right) d \tau .
$$

By Young's convolution inequality we get, for all $a \in[1, \infty)$,

$$
\left\|b_{q}\right\|_{L_{t}^{\infty} L^{p}}+2^{\frac{2 q}{a}}\left\|b_{q}\right\|_{L_{t}^{a} L^{p}} \leqslant c_{a}\left\|b_{q}(0)\right\|_{L^{p}}+c_{a}\left\|\left[\Delta_{q}, v \cdot \nabla\right] b\right\|_{L_{t}^{1} L^{p}}+\left\|F_{q}\right\|_{L_{t}^{1} L^{p}} .
$$

To estimate the commutator in the right hand side, we can use Proposition 2.1.

$$
\left\|\left[\Delta_{q}, v \cdot \nabla\right] b\right\|_{L_{t}^{1} L^{p}} \lesssim \int_{0}^{t}\|b(\tau)\|_{L^{p}}\left((q+2)\|\omega(\tau)\|_{L^{\infty}}+\|v(\tau)\|_{L^{2}}\right) d \tau .
$$

As for the second term in (4.16), we us the continuity of the operator $\Delta_{q}$ in the $L^{p}$ space to write

$$
\left\|F_{q}\right\|_{L_{t}^{1} L^{p}}=\left\|\Delta_{q}\left(\frac{v^{r}}{r} b\right)\right\|_{L_{t}^{1} L^{p}} \lesssim \int_{0}^{t}\left\|\frac{v^{r}}{r}(\tau)\right\|_{L^{\infty}}\|b(\tau)\|_{L^{p}} d \tau .
$$

Inserting the two last estimates into (4.16) we find

$$
\begin{aligned}
&\left\|b_{q}\right\|_{L_{t}^{\infty} L^{p}}+2^{\frac{2 q}{a}}\left\|b_{q}\right\|_{L_{t}^{a} L^{p}} \\
&\left\|b_{q}(0)\right\|_{L^{p}}+\|b\|_{L_{t}^{1} L^{\sigma}}\left(\|v\|_{L_{t}^{\infty} L^{2}}+\left\|v^{r} / r\right\|_{L_{t}^{\infty} L^{\infty}}\right)+(q+2) \int_{0}^{t}\|b(\tau)\|_{L^{p}}\|\omega(\tau)\|_{L^{\infty}} d \tau .
\end{aligned}
$$

Using the last estimate, with $a=1$ and $p=\sigma>3$, we obtain

$$
\begin{aligned}
\|b\|_{L_{t}^{\infty} B_{\sigma, 1}^{\frac{3}{\sigma}-1}}+\|b\|_{L_{t}^{1} B_{\sigma, 1}^{\frac{3}{\sigma}+1}} \lesssim & \left\|\Delta_{-1} b\right\|_{L_{t}^{1} L^{\sigma}}+\left\|b_{0}\right\|_{B_{\sigma, 1}^{\frac{3}{\sigma}-1}}+\|b\|_{L_{t}^{1} L^{\sigma}}\left(\|v\|_{L_{t}^{\infty} L^{2}}+\left\|v^{r} / r\right\|_{L_{t}^{\infty} L^{\infty}}\right)\left(\sum_{q \geqslant 0} 2^{q\left(\frac{3}{\sigma}-1\right)}\right) \\
& +\int_{0}^{t}\|b(\tau)\|_{L^{\sigma}}\|\omega(\tau)\|_{L^{\infty}} d \tau\left(\sum_{q \geqslant 0} 2^{q\left(\frac{3}{\sigma}-1\right)}(q+2)\right) \\
\lesssim & \left\|b_{0}\right\|_{B_{\sigma, 1}^{\frac{3}{\sigma}-1}}+\|b\|_{L_{t}^{1} L^{\sigma}}\left(1+\|v\|_{L_{t}^{\infty} L^{2}}+\left\|v^{r} / r\right\|_{L_{t}^{\infty} L^{\infty}}\right) \\
& +\int_{0}^{t}\|b(\tau)\|_{L^{\sigma}}\|\omega(\tau)\|_{L^{\infty}} d \tau .
\end{aligned}
$$

Then combining (4.15) and (4.18) gives

$$
\begin{aligned}
\|\omega(t)\|_{L^{\infty}} & \leqslant C_{0}+C_{0}\|b\|_{L_{t}^{1} L^{\sigma}}\left(1+\|v\|_{L_{t}^{\infty} L^{2}}+\left\|v^{r} / r\right\|_{L_{t}^{\infty} L^{\infty}}\right) \\
& +C_{0} \int_{0}^{t}\left(\|b(\tau)\|_{L^{\sigma}}+\left\|v^{r}(\tau) / r\right\|_{L^{\infty}}\right)\|\omega(\tau)\|_{L^{\infty}} d \tau .
\end{aligned}
$$

Thanks to Gronwall's inequality we infer that

$$
\|\omega(t)\|_{L^{\infty}} \leqslant C_{0}\left(1+\|b\|_{L_{t}^{1} L^{\sigma}}\left(1+\|v\|_{L_{t}^{\infty} L^{2}}+\left\|v^{r} / r\right\|_{L_{t}^{\infty} L^{\infty}}\right)\right) e^{C_{0}\|b\|_{L_{t}^{1} L^{\sigma}}+C_{0}\left\|v^{r} / r\right\|_{L_{t}^{\infty} L^{\infty}}} .
$$

From Proposition 4.1 and Proposition 4.2 we conclude that

$$
\|\omega(t)\|_{L^{\infty}} \leqslant C_{0}\left(1+\left(t^{\frac{5}{4}}+t^{\frac{1}{4}}\right) \Phi_{1}(t)\right) e^{C_{0}\left(t^{\frac{5}{4}}+t^{\frac{1}{4}}\right)+\Phi_{1}(t)} \leqslant \Phi_{2}(t) .
$$


This gives in turn, by (4.18),

$$
\|b\|_{L_{t}^{\infty} B_{\sigma, 1}^{\frac{3}{\sigma}-1}}+\|b\|_{L_{t}^{1} B_{\sigma, 1}^{\frac{3}{\sigma}+1}} \leqslant \Phi_{2}(t) .
$$

Next we shall prove the estimate on $\|b\|_{L_{t}^{2} L^{\infty}}$. By Bernstein inequality, for $\sigma>3$, combined the estimate (4.17), at $a=2$, we get

$$
\begin{aligned}
\|b\|_{L_{t}^{2} B_{\infty, 1}^{0}} & \leqslant\left\|\Delta_{-1} b\right\|_{L_{t}^{2} L^{\infty}}+\sum_{q \geqslant 0}\left\|\Delta_{q} b\right\|_{L_{t}^{2} L^{\infty}} \\
& \lesssim\|b\|_{L_{t}^{2} L^{2}}+\sum_{q \geqslant 0} 2^{q \frac{3}{\sigma}}\left\|b_{q}\right\|_{L_{t}^{2} L^{\sigma}} \\
& \lesssim\|b\|_{L_{t}^{2} L^{2}}+\left\|b_{0}\right\|_{B_{\sigma, 1}^{\frac{3}{\sigma}-1}}+\|b\|_{L_{t}^{1} L^{\sigma}}\left(\|v\|_{L_{t}^{\infty} L^{2}}+\left\|v^{r} / r\right\|_{L_{t}^{\infty} L^{\infty}}+\|\omega\|_{L_{t}^{\infty} L^{\infty}}\right) \sum_{q \geqslant 0} 2^{q\left(\frac{3}{\sigma}-1\right)}(q+2) \\
& \lesssim\|b\|_{L_{t}^{2} L^{2}}+\left\|b_{0}\right\|_{B_{\sigma, 1}^{\frac{3}{\sigma}-1}}+\|b\|_{L_{t}^{1} L^{\sigma}}\left(\|v\|_{L_{t}^{\infty} L^{2}}+\left\|v^{r} / r\right\|_{L_{t}^{\infty} L^{\infty}}+\|\omega\|_{L_{t}^{\infty} L^{\infty}}\right) .
\end{aligned}
$$

From Proposition 4.1. Proposition 4.2 and the estimate (4.19) we conclude that

$$
\|b\|_{L_{t}^{2} B_{\infty, 1}^{0}} \leqslant C_{0}\left(t^{\frac{1}{2}}+1\right)+C_{0}\left(t^{\frac{5}{4}}+t^{\frac{1}{4}}\right)\left(1+\Phi_{1}(t)+\Phi_{2}(t)\right) \leqslant \Phi_{2}(t) .
$$

This completes the proof of the proposition.

The following global a priori estimate will be needed for the propagation of the critical Besov regularities.

Proposition 4.4. Let $v_{0} \in L^{2} \cap L^{\infty}, b_{0} \in L^{2} \cap B_{\sigma, 1}^{\frac{3}{\sigma}-1}, 3<\sigma<\infty$ be two smooth axisymmetric vector fields with zero divergence such that $\omega_{0} \in L^{\infty}, \omega_{0} / r \in L^{3,1}$ and $b_{0}^{\theta} / r \in L^{2} \cap L^{\infty}$. Then, for every $t \in \mathbb{R}+$,

$$
\|v\|_{L_{t}^{\infty} L^{\infty}} \leqslant \Phi_{3}(t)
$$

Proof. We will use an argument due to Serfati [39] and applied for the Euler case,

$$
\|v(t)\|_{L^{\infty}} \leqslant\left\|\dot{S}_{-N} v(t)\right\|_{L^{\infty}}+\sum_{q \geqslant-N}\left\|\Delta_{q} v(t)\right\|_{L^{\infty}}
$$

where $N$ is an arbitrary positive integer that will be fixed later. By Bernstein inequality we get

$$
\sum_{j \geqslant-N}\left\|\Delta_{q} v(t)\right\|_{L^{\infty}} \lesssim \sum_{q \geqslant-N} 2^{-q}\left\|\Delta_{q} \omega(t)\right\|_{L^{\infty}} \lesssim 2^{N}\|\omega(t)\|_{L^{\infty}}
$$

Using the matrix Leray operator $\mathbb{P}$, the first equation of (1.1) can be reformulated as follows

$$
\partial_{t} v=\mathbb{P}((b \cdot \nabla) b-(v \cdot \nabla) v) .
$$

Therefore, one has the estimate

$$
\begin{aligned}
\left\|\dot{S}_{-N} v\right\|_{L^{\infty}} & \leqslant\left\|\dot{S}_{-N} v_{0}\right\|_{L^{\infty}}+\int_{0}^{t}\left(\left\|\dot{S}_{-N} \mathbb{P}(b \cdot \nabla) b(\tau)\right\|_{L^{\infty}}+\left\|\dot{S}_{-N} \mathbb{P}(v \cdot \nabla) v(\tau)\right\|_{L^{\infty}}\right) d \tau \\
& \leqslant\left\|v_{0}\right\|_{L^{\infty}}+\sum_{q \leqslant-N} \int_{0}^{t}\left(\left\|\dot{\Delta}_{q} \operatorname{div} \mathbb{P}(b \otimes b)(\tau)\right\|_{L^{\infty}}+\left\|\dot{\Delta}_{q} \operatorname{div} \mathbb{P}(v \otimes v)(\tau)\right\|_{L^{\infty}}\right) d \tau \\
& \lesssim\left\|v_{0}\right\|_{L^{\infty}}+\sum_{q \leqslant-N} 2^{q} \int_{0}^{t}\left(\left\|\dot{\Delta}_{q} \mathbb{P}(b \otimes b)(\tau)\right\|_{L^{\infty}}+\left\|\dot{\Delta}_{q} \mathbb{P}(v \otimes v)(\tau)\right\|_{L^{\infty}}\right) d \tau .
\end{aligned}
$$


Since $\dot{\Delta}_{q} \mathbb{P}$ is continuous from $L^{\infty}$ to $L^{\infty}$ we deduce that

$$
\left\|\dot{S}_{-N} v\right\|_{L^{\infty}} \lesssim\left\|v_{0}\right\|_{L^{\infty}}+\int_{0}^{t}\|b(\tau)\|_{L^{\infty}}^{2} d \tau+2^{-N} \int_{0}^{t}\|v(\tau)\|_{L^{\infty}}^{2} d \tau
$$

Inserting (4.22) and (4.24) into (4.21) we obtain

$$
\|v(t)\|_{L^{\infty}} \lesssim\left\|v_{0}\right\|_{L^{\infty}}+\int_{0}^{t}\|b(\tau)\|_{L^{\infty}}^{2} d \tau+2^{N}\|\omega(t)\|_{L^{\infty}}+2^{-N} \int_{0}^{t}\|v(\tau)\|_{L^{\infty}}^{2} d \tau .
$$

We choose $N$ such that

$$
2^{2 N} \approx 1+\|\omega(t)\|_{L^{\infty}}^{-1} \int_{0}^{t}\|v(\tau)\|_{L^{\infty}}^{2} d \tau
$$

we find

$$
\|v(t)\|_{L^{\infty}}^{2} \lesssim\left\|v_{0}\right\|_{L^{\infty}}^{2}+\left(\int_{0}^{t}\|b(\tau)\|_{L^{\infty}}^{2} d \tau\right)^{2}+\|\omega(t)\|_{L^{\infty}}^{2}+\|\omega(t)\|_{L^{\infty}}\left(\int_{0}^{t}\|v(\tau)\|_{L^{\infty}}^{2} d \tau\right) .
$$

According to Gronwall's inequality we get

$$
\begin{aligned}
\|v(t)\|_{L^{\infty}}^{2} & \lesssim\left(\left\|v_{0}\right\|_{L^{\infty}}^{2}+\left(\int_{0}^{t}\|b(\tau)\|_{L^{\infty}}^{2} d \tau\right)^{2}+\|\omega(t)\|_{L^{\infty}}^{2}\right) e^{C t\|\omega\|_{L_{t}^{\infty} L^{\infty}}} \\
& \lesssim\left(\left\|v_{0}\right\|_{L^{\infty}}^{2}+\|b\|_{L_{t}^{2} B_{\infty, 1}^{0}}^{4}+\|\omega(t)\|_{L^{\infty}}^{2}\right) e^{C t\|\omega\|_{L_{t}^{\infty}} L^{\infty}} .
\end{aligned}
$$

where we have used in the last estimate the embedding $B_{\infty, 1}^{0} \hookrightarrow L^{\infty}$. Then, by Proposition 4.3 we conclude that

$$
\|v(t)\|_{L^{\infty}}^{2} \leqslant \Phi_{3}(t)
$$

which is the desired estimate.

\section{$5 \quad$ Sub-critical regularities}

In this section we intend to prove Theorem 1.1. We first deal with some a priori estimates. In a second step we we highlight the proof of the existence part and finally we give the uniqueness result.

\subsection{Lipschitz bound of the velocity}

We shall prove the global propagation of the sub-critical Sobolev regularities, which is heavily related to the control of the Lipschitz norm of the velocity. The main result of this section is the following:

Proposition 5.1. Let $v_{0} \in H^{s}$ and $b_{0} \in H^{s-2}, s>5 / 2$, be two divergence-free axisymmetric vector field as in (3.1). Assume in addition that $b_{0}^{\theta} / r \in L^{2} \cap L^{\infty}$ Then any smooth solution $(u, v)$ of the MHD system (1.1) satisfies

$$
\|v\|_{\widetilde{L}_{t}^{\infty} H^{s}}+\|b\|_{\widetilde{L}_{t}^{\infty} H^{s-2}}+\|b\|_{\widetilde{L}_{t}^{1} H^{s}} \leqslant \Phi_{5}(t) .
$$

Proof. We localize in frequency the equation of the velocity in (1.1). For $q \in \mathbb{N} \cup\{-1\}$ we set $v_{q}:=\Delta_{q} v$ and $b_{q}:=\Delta_{q} b$. Then, we have

$$
\partial_{t} v_{q}+v \cdot \nabla v_{q}+\nabla p_{q}=\Delta_{q}(b \cdot \nabla b)-\left[\Delta_{q}, v \cdot \nabla\right] v .
$$


Taking the $L^{2}$-inner product with $v_{q}$ and using the incompressibility of $v$ and $v_{q}$ we get

$$
\frac{d}{d t}\left\|v_{q}(t)\right\|_{L^{2}} \leqslant\left\|\Delta_{q}(b \cdot \nabla b)\right\|_{L^{2}}+\left\|\left[\Delta_{q}, v \cdot \nabla\right] v\right\|_{L^{2}} .
$$

Then integrating in time gives

$$
\left\|v_{q}(t)\right\|_{L^{2}} \leqslant\left\|v_{q}(0)\right\|_{L^{2}}+\left\|\Delta_{q}(b \cdot \nabla b)\right\|_{L_{t}^{1} L^{2}}+\left\|\left[\Delta_{q}, v \cdot \nabla\right] v\right\|_{L_{t}^{1} L^{2}} .
$$

Multiplying this last inequality by $2^{q s}$, then taking the $\ell^{2}$-norm we find

$$
\|v\|_{\widetilde{L}_{t}^{\infty} H^{s}} \lesssim\left\|v_{0}\right\|_{H^{s}}+\|b \cdot \nabla b\|_{\widetilde{L}_{t}^{1} H^{s}}+\left\|\left(2^{q s}\left\|\left[\Delta_{q}, v \cdot \nabla\right] v\right\|_{L_{t}^{1} L^{2}}\right)_{q}\right\|_{\ell^{2}} .
$$

Next we shall make use of the following commutator estimate, see for instance Lemma B.5 in [18],

$$
\left\|\left(2^{q s}\left\|\left[\Delta_{q}, v \cdot \nabla\right] v\right\|_{L_{t}^{1} L^{2}}\right)_{q}\right\|_{\ell^{2}} \lesssim \int_{0}^{t}\|\nabla v(\tau)\|_{L^{\infty}}\|v(\tau)\|_{H^{s}} d \tau .
$$

Moreover, since $\operatorname{curl}(b \cdot \nabla b)=-\partial_{z}\left(\frac{b^{\theta}}{r} b\right)$ then by Proposition A.1 we get

$$
\begin{aligned}
\|b \cdot \nabla b\|_{\widetilde{L}_{t}^{1} H^{s}} & \leqslant\|b \cdot \nabla b\|_{\tilde{L}_{t}^{1} L^{2}}+\|\operatorname{curl}(b \cdot \nabla b)\|_{\widetilde{L}_{t}^{1} H^{s-1}} \\
& \lesssim\|b\|_{\widetilde{L}_{t}^{\infty} L^{2}}\|\nabla b\|_{L_{t}^{1} L^{\infty}}+\left\|\frac{b^{\theta}}{r} b\right\|_{\widetilde{L}_{t}^{1} H^{s}} \\
& \lesssim\|b\|_{L_{t}^{\infty} L^{2}}\|\nabla b\|_{L_{t}^{1} L^{\infty}}+\left\|\frac{b^{\theta}}{r}\right\|_{L_{t}^{\infty} L^{\infty}}\|b\|_{\widetilde{L}_{t}^{1} H^{s}} .
\end{aligned}
$$

This together with (5.2) and (5.1) gives

$$
\|v\|_{\widetilde{L}_{t}^{\infty} H^{s}} \lesssim\left\|v_{0}\right\|_{H^{s}}+\|b\|_{L_{t}^{\infty} L^{2}}\|\nabla b\|_{L_{t}^{1} L^{\infty}}+\left\|\frac{b^{\theta}}{r}\right\|_{L_{t}^{1} L^{\infty}}\|b\|_{\widetilde{L}_{t}^{1} H^{s}}+\int_{0}^{t}\|\nabla v(\tau)\|_{L^{\infty}}\|v(\tau)\|_{H^{s}} d \tau .
$$

Next, we shall estimate $\|b\|_{\widetilde{L}_{t}^{1} H^{s}}$. According to (4.16), one has

$$
\left\|b_{q}\right\|_{L_{t}^{\infty} L^{2}}+2^{2 q}\left\|b_{q}\right\|_{L_{t}^{1} L^{2}} \lesssim\left\|b_{q}(0)\right\|_{L^{2}}+\left\|\Delta_{q}(b \cdot \nabla v)\right\|_{L_{t}^{1} L^{2}}+\left\|\left[\Delta_{q}, v \cdot \nabla\right] b(\tau)\right\|_{L_{t}^{1} L^{2}}
$$

It follows that

$\|b\|_{\widetilde{L}_{t}^{\infty} H^{s-2}}+\|b\|_{\widetilde{L}_{t}^{1} H^{s}} \lesssim\left\|b_{0}\right\|_{H^{s-2}}+\left\|\Delta_{-1} b\right\|_{L_{t}^{1} L^{2}}+\|b \cdot \nabla v\|_{\widetilde{L}_{t}^{1} H^{s-2}}+\left\|\left(2^{q(s-2)}\left\|\left[\Delta_{q}, v \cdot \nabla\right] b(\tau)\right\|_{L_{t}^{1} L^{2}}\right)_{q}\right\|_{\ell^{2}}$.

Since $s-2>\frac{1}{2}$ then $H^{s-2} \cap L^{\infty}$ is an algebra and, by Lemma 2.2, we get

$$
\|b \cdot \nabla v\|_{\widetilde{L}_{t}^{1} H^{s-2}} \lesssim\|b \cdot \nabla v\|_{L_{t}^{1} H^{s-2}} \lesssim \int_{0}^{t}\left(\|b(\tau)\|_{H^{s-2}}\|\nabla v(\tau)\|_{L^{\infty}}+\|b(\tau)\|_{L^{\infty}}\|v(\tau)\|_{H^{s-1}}\right) d \tau
$$

Now, in order to estimate the commutator term, we shall use the estimate, (see for instance Lemma 2.100 in [4])

$$
\left\|\left(2^{q(s-2)}\left\|\left[\Delta_{q}, v \cdot \nabla\right] b\right\|_{L_{t}^{1} L^{2}}\right)_{q}\right\|_{\ell^{2}} \lesssim \int_{0}^{t}\left(\|\nabla v(\tau)\|_{L^{\infty}}\|b(\tau)\|_{H^{s-2}}+\|\nabla b(\tau)\|_{L^{\infty}}\|v(\tau)\|_{H^{s-2}}\right) d \tau .
$$


Combining the three last inequalities and using the embedding $H^{s} \hookrightarrow H^{s-2}$ we deduce that

$$
\begin{aligned}
& \|b\|_{\tilde{L}_{t}^{\infty} H^{s-2}}+\|b\|_{\widetilde{L}_{t}^{1} H^{s}} \lesssim\left\|b_{0}\right\|_{H^{s-2}}+\|b\|_{L_{t}^{1} L^{2}} \\
& +\int_{0}^{t}\left(\|\nabla v(\tau)\|_{L^{\infty}}\|b(\tau)\|_{H^{s-2}}+\left(\|\nabla b(\tau)\|_{L^{\infty}}+\|b(\tau)\|_{L^{\infty}}\right)\|v(\tau)\|_{H^{s}}\right) d \tau .
\end{aligned}
$$

Putting together (5.3) and (5.4) and using Proposition 4.1 we find

$$
\begin{aligned}
\|v\|_{\tilde{L}_{t}^{\infty} H^{s}}+\|b\|_{\widetilde{L}_{t}^{\infty} H^{s-2}}+\|b\|_{\tilde{L}_{t}^{1} H^{s}} \leqslant & C_{0}\left(1+t+\|\nabla b\|_{L_{t}^{1} L^{\infty}}\right) \\
& +C_{0} \int_{0}^{t}\|\nabla v(\tau)\|_{L^{\infty}}\left(\|b(\tau)\|_{H^{s-2}}+\|v(\tau)\|_{H^{s}}\right) d \tau \\
& +C_{0} \int_{0}^{t}\left(\|\nabla b(\tau)\|_{L^{\infty}}+\|b(\tau)\|_{L^{\infty}}\right)\|v(\tau)\|_{H^{s}} d \tau .
\end{aligned}
$$

Then, an application of the Gronwall inequality gives rise to

$$
\|v\|_{\tilde{L}_{t}^{\infty} H^{s}}+\|b\|_{\tilde{L}_{t}^{\infty} H^{s-2}}+\|b\|_{\widetilde{L}_{t}^{1} H^{s}} \leqslant C_{0}(t+1) e^{C_{0}\left(\|\nabla v\|_{L_{t}^{1} L^{\infty}}+\|\nabla b\|_{L_{t}^{1} L^{\infty}}+\|b\|_{L_{t}^{1} L^{\infty}}\right)} .
$$

By Proposition 4.17(iii), the embeddings

$$
\begin{aligned}
& H^{s-2} \hookrightarrow L^{\sigma} \hookrightarrow B_{\sigma, 1}^{\frac{3}{\sigma}-1}, \\
& B_{\sigma, 1}^{\frac{3}{\sigma}+1} \hookrightarrow \operatorname{Lip}\left(\mathbb{R}^{3}\right),
\end{aligned}
$$

for some $\sigma>3$, and Proposition 4.3 we deduce that

$$
\|v\|_{\widetilde{L}_{t}^{\infty} H^{s}}+\|b\|_{\widetilde{L}_{t}^{\infty} H^{s-2}}+\|b\|_{\widetilde{L}_{t}^{1} H^{s}} \leqslant \Phi_{3}(t) e^{C\|\nabla v\|_{L_{t}^{1} L^{\infty}}} .
$$

Now, to get the global bound for the Lipschitz norm of the velocity we use the classical logarithmic estimate: for $s>\frac{5}{2}$,

$$
\|\nabla v\|_{L^{\infty}} \lesssim\|v\|_{L^{2}}+\|\omega\|_{L^{\infty}} \log \left(e+\|v\|_{H^{s}}\right) .
$$

Therefore, by (5.5) and Proposition 4.3,

$$
\|\nabla v\|_{L^{\infty}} \leqslant \Phi_{3}(t)\left(1+\int_{0}^{t}\|\nabla v(\tau)\|_{L^{\infty}}\right) .
$$

By Gronwall's inequality we get

$$
\|\nabla v\|_{L^{\infty}} \leqslant \Phi_{4}(t)
$$

Plugging this estimate into (5.5) we conclude that

$$
\|v\|_{\widetilde{L}_{t}^{\infty} H^{s}}+\|b\|_{\widetilde{L}_{t}^{\infty} H^{s-2}}+\|b\|_{\widetilde{L}_{t}^{1} H^{s}} \leqslant \Phi_{5}(t) .
$$

This completes the proof of the proposition. 


\subsection{Existence}

We will briefly outline the proof of the existence part which is classical. We smooth out the initial data

$$
v_{0}^{n}=S_{n} v^{0}=2^{3 n} g\left(2^{n} .\right) * v_{0} \quad \text { and } \quad b_{0}^{n}=S_{n} b^{0}=2^{3 n} g\left(2^{n} .\right) * b_{0} .
$$

where $S_{n}$ is the cut-off frequency defined in Section 2. Because $g$ is radial then the functions $v_{0}^{n}$ and $b_{0}^{n}$ remain axisymmetric. Moreover, this family is uniformly bounded in the space of initial data. Then, by using standard arguments based on the a priori estimates given by Proposition 4.1, Proposition 4.2. Proposition 4.3 and Proposition 5.1 we can construct a unique global solution $\left(v_{n}, b_{n}\right)_{n \in \mathbb{N}}$ in the following space

$$
v_{n} \in \mathcal{C}\left(\mathbb{R}_{+} ; L^{2}\right) \cap L^{1}\left(\mathbb{R}_{+} ; W^{1, \infty}\right) \quad \text { and } \quad b_{n} \in \mathcal{C}\left(\mathbb{R}_{+} ; H^{s-2}\right) \cap L^{1}\left(\mathbb{R}_{+} ; W^{1, \infty}\right) .
$$

The control is uniform with respect to the parameter $n$. Therefore by standard compactness arguments we can show that this family $\left(v_{n}, b_{n}\right)_{n \in \mathbb{N}}$ converges to some $(v, b)$ which satisfies our initial value problem. We omit here the details and we will next focus on the uniqueness part.

\subsection{Uniqueness result}

We shall prove the uniqueness result for the system (1.1) in the following space

$$
(v, b) \in \mathcal{X}:=\left(\mathcal{C}\left(\mathbb{R}_{+}, L^{2}\right) \cap L^{1}\left(\mathbb{R}_{+} ; W^{1, \infty}\right)\right)^{2} .
$$

We take two solutions $\left(v_{j}, b_{j}\right)$, with $j=1,2$ for (1.1) belonging to the space $\mathcal{X}$ with initial data $\left(v_{j}^{0}, b_{j}^{0}\right), j=1,2$ and we denote

$$
v=v_{2}-v_{1} \quad \text { and } \quad b=b_{2}-b_{1} .
$$

Then we find the equations

$$
\left\{\begin{array}{l}
\partial_{t} v+v_{2} \cdot \nabla v+\nabla p=-v \cdot \nabla v_{1}+b_{2} \cdot \nabla b+b \cdot \nabla b_{1}, \\
\partial_{t} b+v_{2} \cdot \nabla b-\Delta b=-v \cdot \nabla b_{1}+b_{2} \cdot \nabla v+b \cdot \nabla v_{1}, \\
v_{\mid t=0}=v^{0}, \quad b_{\mid t=0}=b^{0}
\end{array}\right.
$$

Taking the $L^{2}$ inner product of the first equation of (5.6) with $v$ and integrating by parts,

$$
\frac{1}{2} \frac{d}{d t}\|v(t)\|_{L^{2}}^{2}=-\int_{\mathbb{R}^{3}}\left(v \cdot \nabla v_{1}\right) \cdot v d x-\int_{\mathbb{R}^{3}}\left(b_{2} \cdot \nabla v\right) \cdot b d x-\int_{\mathbb{R}^{3}}(b \cdot \nabla v) \cdot b_{1} d x
$$

Taking the $L^{2}$ inner product of the second equation of (5.6) with $b$ we get

$$
\frac{1}{2} \frac{d}{d t}\|b(t)\|_{L^{2}}^{2}+\int_{\mathbb{R}^{3}}|\nabla b(t, x)| \cdot b_{q} d x=-\int_{\mathbb{R}^{3}}\left(v \cdot \nabla b_{1}\right) \cdot b d x+\int_{\mathbb{R}^{3}}\left(b_{2} \cdot \nabla v\right) \cdot b d x+\int_{\mathbb{R}^{3}}(b \cdot \nabla v) \cdot b_{1} d x .
$$

Summing the two last estimate and using Hölder and Young inequalities, we get

$$
\frac{1}{2} \frac{d}{d t}\left(\|v(t)\|_{L^{2}}^{2}+\|b(t)\|_{L^{2}}^{2}\right) \leqslant\left(\left\|\nabla v_{1}(t)\right\|_{L^{\infty}}+\left\|\nabla \rho_{1}(t)\right\|_{L^{\infty}}\right)\left(\|v(t)\|_{L^{2}}^{2}+\|b(t)\|_{L^{2}}^{2}\right) .
$$

Gronwall's inequality yields

$$
\|v(t)\|_{L^{2}}^{2}+\|b(t)\|_{L^{2}}^{2} \leqslant\left(\left\|v_{0}\right\|_{L^{2}}^{2}+\left\|b_{0}\right\|_{L^{2}}^{2}\right) \exp \left(2\left(\left\|\nabla v_{1}(t)\right\|_{L_{t}^{1} L^{\infty}}+\left\|\nabla \rho_{1}(t)\right\|_{L_{t}^{1} L^{\infty}}\right)\right) .
$$

This proves the uniqueness result. 


\section{Critical regularities}

This section is devoted to the proof of Theorem 1.2. We restrict ourselves to the a priori estimates. The existence and uniqueness parts can be easily obtained in a classical way.

\subsection{Vorticity decomposition}

In order to get a global bound on the quantity $\|\omega(t)\|_{B_{\infty, 1}^{0},}$, we shall use the approach developed in the 3D axisymmetric Euler case in [3]. We write a decomposition of the vorticity based on the special structure of axisymmetric flows. This later one is the basic tool to get the Lipschitz bound of the velocity.

Proposition 6.1. There exists a decomposition $\left(\widetilde{\omega}_{j}\right)_{j \geqslant-1}$ of $\omega$ such that

(i) For every $t \in \mathbb{R}_{+}, \omega(t, x)=\sum_{j \geqslant-1} \widetilde{\omega}_{j}(t, x)$.

(ii) For every $t \in \mathbb{R}_{+}, j \geqslant-1$, div $\tilde{\omega}_{j}(t, x)=0$.

(iii) For all $j \geqslant-1$ we have

$$
\left\|\widetilde{\omega}_{j}(t)\right\|_{L^{\infty}} \leqslant C\left(\left\|\Delta_{j} \omega^{0}\right\|_{L^{\infty}}+2^{j}\left\|\Delta_{j}\left(\frac{b^{\theta}}{r} b_{1}\right)\right\|_{L_{t}^{1} L^{\infty}}\right) \Phi_{2}(t) .
$$

(iv) For all $k, j \geqslant-1$ we have

$$
\left\|\Delta_{k} \widetilde{\omega}_{j}(t)\right\|_{L^{\infty}} \lesssim 2^{-|k-j|} e^{C V(t)}\left(\left\|\Delta_{j} \omega^{0}\right\|_{L^{\infty}}+2^{j}\left\|\Delta_{j}\left(\frac{b^{\theta}}{r} b\right)\right\|_{L_{t}^{1} L^{\infty}}\right),
$$

with $V(t):=\|v(t)\|_{L_{t}^{1} B_{\infty, 1}^{1}}$.

Proof. Denote by $\left(\widetilde{\omega}_{j}\right)_{j \geqslant-1}$ the unique global solution of the equation,

$$
\left\{\begin{array}{l}
\partial_{t} \widetilde{\omega}_{j}+v \cdot \nabla \widetilde{\omega}_{j}=\widetilde{\omega}_{j} . \nabla v-\partial_{z}\left[\Delta_{j}\left(\frac{b^{\theta}}{r} b\right)\right], \\
\widetilde{\omega}_{j}(t=0)=\Delta_{j} \omega^{0} .
\end{array}\right.
$$

Since $\operatorname{div}\left(\Delta_{j} \omega^{0}\right)=0$ then from Proposition 3.1 (i) we have

$$
\operatorname{div} \widetilde{\omega}_{j}(t)=0 .
$$

By linearity and uniqueness we have

$$
\omega(t, x)=\sum_{j \geqslant-1} \widetilde{\omega}_{j}(t, x)
$$

Next we shall prove the estimate in (iii). Since $\Delta_{j} \omega^{0}=\operatorname{curl}\left(\Delta_{j} v^{0}\right)$ and $\Delta_{j} v^{0}$ is axisymmetric then Proposition 3.1 implies that $\Delta_{j} \omega^{0} \times e_{\theta}=(0,0,0)$. Therefore, by Proposition 3.2 we get $\widetilde{\omega}_{j}(t) \times e_{\theta}=$ $(0,0,0)$ and

$$
\partial_{t} \widetilde{\omega}_{j}+v \cdot \nabla \widetilde{\omega}_{j}=\widetilde{\omega}_{j} \frac{v^{r}}{r}-\partial_{z}\left[\Delta_{j}\left(\frac{b^{\theta}}{r} b\right)\right] .
$$


By the maximum principle, Propositions 4.2 and Bernstein inequality we get

$$
\begin{aligned}
\left\|\widetilde{\omega}_{j}(t)\right\|_{L^{\infty}} & \leqslant\left\|\Delta_{j} \omega^{0}\right\|_{L^{\infty}}+\int_{0}^{t}\left\|\frac{v^{r}(\tau)}{r}\right\|_{L^{\infty}}\left\|\widetilde{\omega}_{j}(\tau)\right\|_{L^{\infty}} d \tau+\int_{0}^{t}\left\|\partial_{z}\left[\Delta_{j}\left(\frac{b^{\theta}}{r} b\right)(\tau)\right]\right\|_{L^{\infty}} d \tau \\
& \lesssim\left\|\Delta_{j} \omega^{0}\right\|_{L^{\infty}}+\int_{0}^{t} \Phi_{1}(\tau)\left\|\widetilde{\omega}_{j}(\tau)\right\|_{L^{\infty}} d \tau+2^{j} \int_{0}^{t}\left\|\Delta_{j}\left(\frac{b^{\theta}}{r} b\right)(\tau)\right\|_{L^{\infty}} d \tau .
\end{aligned}
$$

Then, the Gronwall's inequality implies that

$$
\left\|\widetilde{\omega}_{j}(t)\right\|_{L^{\infty}} \leqslant C\left(\left\|\Delta_{j} \omega^{0}\right\|_{L^{\infty}}+2^{j}\left\|\Delta_{j}\left(\frac{b^{\theta}}{r} b\right)\right\|_{L_{t}^{1} L^{\infty}}\right) \Phi_{2}(t) .
$$

This concludes (iii).

In order to prove (iv), we shall equivalently show that

$$
\left\|\Delta_{k} \widetilde{\omega}_{j}(t)\right\|_{L^{\infty}} \lesssim 2^{k-j} e^{C V(t)}\left(\left\|\Delta_{j} \omega^{0}\right\|_{L^{\infty}}+2^{j}\left\|\Delta_{j}\left(\frac{b^{\theta}}{r} b\right)\right\|_{L_{t}^{1} L^{\infty}}\right)
$$

and

$$
\left\|\Delta_{k} \widetilde{\omega}_{j}(t)\right\|_{L^{\infty}} \lesssim 2^{j-k} e^{C V(t)}\left(\left\|\Delta_{j} \omega^{0}\right\|_{L^{\infty}}+2^{j}\left\|\Delta_{j}\left(\frac{b^{\theta}}{r} b\right)\right\|_{L_{t}^{1} L^{\infty}}\right) .
$$

Proof of (6.2). Applying Proposition 2.3 to the equation (6.1) yields

$$
\begin{aligned}
e^{-C V(t)}\left\|\widetilde{\omega}_{j}(t)\right\|_{B_{\infty, \infty}^{-1}} & \lesssim\left\|\Delta_{j} \omega^{0}\right\|_{B_{\infty}^{-1}, \infty}+\int_{0}^{t} e^{-C V(\tau)}\left\|\widetilde{\omega}_{j} . \nabla v(\tau)\right\|_{B_{\infty, \infty}^{-1}} d \tau \\
& +\int_{0}^{t} e^{-C V(\tau)}\left\|\partial_{z}\left[\Delta_{j}\left(\frac{b^{\theta}}{r} b\right)(\tau)\right]\right\|_{B_{\infty, \infty}^{-1}} d \tau
\end{aligned}
$$

where $V(t)=\int_{0}^{t}\|v(\tau)\|_{B_{\infty, 1}^{1}} d \tau$. Using Bony's decomposition we can easily check that

$$
\left\|\widetilde{\omega}_{j} . \nabla v\right\|_{B_{\infty, \infty}^{-1}} \lesssim\|v\|_{B_{\infty, 1}^{1} \|}\left\|\widetilde{\omega}_{j}\right\|_{B_{\infty, \infty}^{-1}} \cdot
$$

On the other hand, by using Hölder and Bernstein inequalities we obtain

$$
\begin{aligned}
\int_{0}^{t} e^{-C V(\tau)}\left\|\partial_{z}\left[\Delta_{j}\left(\frac{b^{\theta}}{r} b\right)\right](\tau)\right\|_{B_{\infty, \infty}^{-1}} d \tau & \leqslant\left\|\partial_{z}\left[\Delta_{j}\left(\frac{b^{\theta}}{r} b\right)\right]\right\|_{L_{t}^{1} B_{\infty, \infty}^{-1}} \\
& \lesssim\left\|\Delta_{j}\left(\frac{b^{\theta}}{r} b\right)\right\|_{L_{t}^{1} B_{\infty, \infty}^{0}} \\
& \lesssim\left\|\Delta_{j}\left(\frac{b^{\theta}}{r} b\right)\right\|_{L_{t}^{1} L^{\infty}} .
\end{aligned}
$$

Inserting the two last estimates into (6.4) yields,

$$
e^{-C V(t)}\left\|\widetilde{\omega}_{j}(t)\right\|_{B_{\infty, \infty}^{-1}} \lesssim\left\|\Delta_{j} \omega^{0}\right\|_{B_{\infty, \infty}^{-1}}+\left\|\Delta_{j}\left(\frac{b^{\theta}}{r} b\right)\right\|_{L_{t}^{1} L^{\infty}}+\int_{0}^{t} e^{-C V(\tau)}\left\|\widetilde{\omega}_{j}\right\|_{B_{\infty, \infty}^{-1}}\|v(\tau)\|_{B_{\infty, 1}^{1}} d \tau .
$$

Using Gronwall's inequality gives

$$
\begin{aligned}
\left\|\widetilde{\omega}_{j}(t)\right\|_{B_{\infty, \infty}^{-1}} & \lesssim\left(\left\|\Delta_{j} \omega^{0}\right\|_{B_{\infty, \infty}^{-1}}+\left\|\Delta_{j}\left(\frac{b^{\theta}}{r} b\right)\right\|_{L_{t}^{1} L^{\infty}}\right) e^{C V(t)} \\
& \lesssim\left(2^{-j}\left\|\Delta_{j} \omega^{0}\right\|_{L^{\infty}}+\left\|\Delta_{j}\left(\frac{b^{\theta}}{r} b\right)\right\|_{L_{t}^{1} L^{\infty}}\right) e^{C V(t)}
\end{aligned}
$$


Finally, by definition, we deduce that

$$
\left\|\Delta_{k} \widetilde{\omega}_{j}(t)\right\|_{L^{\infty}} \lesssim 2^{k-j} e^{C V(t)}\left(\left\|\Delta_{j} \omega^{0}\right\|_{L^{\infty}}+2^{j}\left\|\Delta_{j}\left(\frac{b^{\theta}}{r} b\right)\right\|_{L_{t}^{1} L^{\infty}}\right) .
$$

which is the desired result.

Proof of (6.3). In this case it is more convenient to work with the cartesian coordinates of $\widetilde{\omega}_{j}=\left(\widetilde{\omega}_{j}^{1}, \widetilde{\omega}_{j}^{2}, 0\right)$. The analysis will be exactly the same for both components, so we will deal only with the first one. Since $v^{\theta}=0$ the one may easily check that

$$
\frac{v^{r}}{r}=\frac{v^{1}}{x_{1}}=\frac{v^{2}}{x_{2}}
$$

Then, the functions $\widetilde{\omega}_{j}^{1}$ solves the equation

$$
\left\{\begin{array}{l}
\partial_{t} \widetilde{\omega}_{j}^{1}+v \cdot \nabla \widetilde{\omega}_{j}^{1}=v^{2} \frac{\widetilde{\omega}_{j}^{1}}{x_{2}}-\partial_{z}\left[\Delta_{j}\left(\frac{b^{\theta}}{r} b_{1}\right)\right], \\
\widetilde{\omega}_{j}^{1}(t=0)=\Delta_{j} \omega_{0}^{1} .
\end{array}\right.
$$

Applying Proposition 2.3 to this last equation we get

$$
\begin{aligned}
e^{-C V(t)}\left\|\widetilde{\omega}_{j}^{1}(t)\right\|_{B_{\infty, 1}^{1}} & \lesssim\left\|\Delta_{j} \omega_{0}^{1}\right\|_{B_{\infty, 1}^{1}}+\int_{0}^{t} e^{-C V(\tau)}\left\|v^{2} \frac{\widetilde{\omega}_{j}^{1}(\tau)}{x_{2}}\right\|_{B_{\infty, 1}^{1}} d \tau \\
& +\int_{0}^{t} e^{-C V(\tau)}\left\|\partial_{z}\left[\Delta_{j}\left(\frac{b^{\theta}}{r} b_{1}\right)\right](\tau)\right\|_{B_{\infty, 1}^{1}} d \tau .
\end{aligned}
$$

We shall make use of the following estimate, proved in pages 31-32 from [3]:

$$
\left\|v^{2} \frac{\widetilde{\omega}_{j}^{1}}{x_{2}}\right\|_{B_{\infty, 1}^{1}} \lesssim\|v\|_{B_{\infty, 1}^{1}}\left(\left\|\widetilde{\omega}_{j}^{1}\right\|_{B_{\infty, 1}^{1}}+\left\|\frac{\widetilde{\omega}_{j}^{1}}{x_{2}}\right\|_{B_{\infty, 1}^{0}}\right) .
$$

Now we will estimate $\left\|\frac{\widetilde{\omega}_{j}^{1}}{x_{2}}\right\|_{L_{t}^{\infty} B_{\infty, 1}^{0}}$. By Proposition 3.1 we have $\widetilde{\omega}_{j}^{1}\left(x_{1}, 0, z\right)=0$. Then, by Taylor formula we get

$$
\frac{\widetilde{\omega}_{j}^{1}\left(x_{1}, x_{2}, z\right)}{x_{2}}=\int_{0}^{1} \partial_{x_{2}} \widetilde{\omega}_{j}^{1}\left(x_{1}, \tau x_{2}, z\right) d \tau .
$$

At this stage we need to the following estimate, describing the anisotropic dilatation in Besov space $B_{\infty, 1}^{0}$, see Proposition A.1 from [3]: for every $0<\tau<1$, we have

$$
\left\|h_{\tau}\right\|_{B_{\infty, 1}^{0}} \leqslant C(1-\log \tau)\|h\|_{B_{\infty, 1}^{0}},
$$

where $h_{\tau}\left(x_{1}, x_{2}, x_{3}\right)=h\left(x_{1}, \tau x_{2}, x_{3}\right)$ and $C$ is a absolute positive constant. It follows that

$$
\left\|\frac{\widetilde{\omega}_{j}^{1}}{x_{2}}\right\|_{B_{\infty, 1}^{0}} \leqslant\left\|\partial_{x_{2}} \widetilde{\omega}_{j}^{1}\right\|_{B_{\infty, 1}^{0}} \int_{0}^{1}(1-\log \tau) d \tau \lesssim\left\|\widetilde{\omega}_{j}^{1}\right\|_{B_{\infty, 1}^{1}} .
$$

Inserting this estimate into (6.7) gives

$$
\left\|v^{2} \frac{\widetilde{\omega}_{j}^{1}}{x_{2}}\right\|_{B_{\infty, 1}^{1}} \lesssim\|v\|_{B_{\infty, 1}^{1}}\left\|\widetilde{\omega}_{j}^{1}\right\|_{B_{\infty, 1}^{1}} .
$$


On the other hand, using Bernstein inequalities we find

$$
\begin{aligned}
\int_{0}^{t} e^{-C V(\tau)}\left\|\partial_{z}\left[\Delta_{j}\left(\frac{b^{\theta}}{r} b_{1}\right)\right](\tau)\right\|_{B_{\infty, 1}^{1}} d \tau & \leqslant\left\|\partial_{z}\left[\Delta_{j}\left(\frac{b^{\theta}}{r} b_{1}\right)\right]\right\|_{L_{t}^{1} B_{\infty, 1}^{1}} \\
& \lesssim\left\|\Delta_{j}\left(\frac{b^{\theta}}{r} b_{1}\right)\right\|_{L_{t}^{1} B_{\infty, 1}^{2}} \\
& \lesssim 2^{2 j}\left\|\Delta_{j}\left(\frac{b^{\theta}}{r} b_{1}\right)\right\|_{L_{t}^{1} L^{\infty}} .
\end{aligned}
$$

Plugging the two last estimates into (6.6) we conclude that

$$
e^{-C V(t)}\left\|\widetilde{\omega}_{j}^{1}(t)\right\|_{B_{\infty, 1}^{1}} \lesssim\left\|\Delta_{j} \omega_{0}^{1}\right\|_{B_{\infty, 1}^{1}}+\int_{0}^{t} e^{-C V(\tau)}\left\|\widetilde{\omega}_{j}^{1}(\tau)\right\|_{B_{\infty, 1}^{1}}\|v(\tau)\|_{B_{\infty, 1}^{1}} d \tau+2^{2 j}\left\|\Delta_{j}\left(\frac{b^{\theta}}{r} b_{1}\right)\right\|_{L_{t}^{1} L^{\infty}} .
$$

Then Gronwall's inequality implies that

$$
\left\|\widetilde{\omega}_{j}^{1}(t)\right\|_{B_{\infty, 1}^{1}} \lesssim\left(2^{j}\left\|\Delta_{j} \omega_{0}\right\|_{L^{\infty}}+2^{2 j}\left\|\Delta_{j}\left(\frac{b^{\theta}}{r} b_{1}\right)\right\|_{L_{t}^{1} L^{\infty}}\right) e^{C V(t)} .
$$

This can be written as

$$
\left\|\Delta_{k} \widetilde{\omega}_{j}^{1}(t)\right\|_{L^{\infty}} \lesssim 2^{j-k} e^{C V(t)}\left(\left\|\Delta_{j} \omega_{0}\right\|_{L^{\infty}}+2^{j}\left\|\Delta_{j}\left(\frac{b^{\theta}}{r} b_{1}\right)\right\|_{L_{t}^{1} L^{\infty}}\right),
$$

ending the proof of the proposition.

\subsection{Lipschitz bound}

The following result deals with the global regularity persistence for initial data belonging to the critical Besov space $B_{\infty, 1}^{1}$.

Proposition 6.2. Let $v_{0} \in L^{2} \cap B_{\infty, 1}^{1}$ such that $\omega_{0}^{\theta} / r \in L^{3,1}$ and $b_{0} \in L^{2} \cap B_{\sigma, 1}^{\frac{3}{\sigma}}, \sigma \in(3, \infty)$, such that $b_{0}^{\theta} / r \in L^{2} \cap L^{\infty}$. Then for every $t \in \mathbb{R}_{+}$,

$$
\|\omega(t)\|_{B_{\infty, 1}^{0}}+\|v(t)\|_{B_{\infty, 1}^{1}} \leqslant \Phi_{4}(t) .
$$

Proof. Let $N$ be a fixed positive integer that will be chosen later. By definition of Besov spaces and in view of Proposition 6.1 (i) one has

$$
\begin{aligned}
\|\omega(t)\|_{B_{\infty, 1}^{0}} & \leqslant \sum_{k}\left\|\Delta_{k} \sum_{j} \widetilde{\omega}_{j}(t)\right\|_{L^{\infty}} \\
& \lesssim \sum_{k}\left(\sum_{|k-j| \geqslant N}\left\|\Delta_{k} \widetilde{\omega}_{j}(t)\right\|_{L^{\infty}}\right)+\sum_{k}\left(\sum_{|k-j|<N}\left\|\Delta_{k} \widetilde{\omega}_{j}(t)\right\|_{L^{\infty}}\right) \\
& :=\mathrm{I}_{1}+\mathrm{I}_{2} .
\end{aligned}
$$

To estimate the term $I_{1}$ we use Proposition 6.1 (iv),

$$
\begin{aligned}
\mathrm{I}_{1} & =\sum_{k}\left(\sum_{|k-j| \geqslant N}\left\|\Delta_{k} \widetilde{\omega}_{j}(t)\right\|_{L^{\infty}}\right) \\
& \lesssim 2^{-N}\left(\left\|\omega_{0}\right\|_{B_{\infty, 1}^{0}}+\left\|\frac{b^{\theta}}{r} b\right\|_{L_{t}^{1} B_{\infty, 1}^{1}}\right) e^{C V(t)} .
\end{aligned}
$$


For the second term $\mathrm{I}_{2}$ we use the continuity of the operator $\Delta_{k}$ in the space $L^{\infty}$ and Proposition 6.1.(iii),

$$
\begin{aligned}
\mathrm{I}_{2} & \lesssim \sum_{j} \sum_{|k-j|<N}\left\|\widetilde{\omega}_{j}(t)\right\|_{L^{\infty}} \\
& \lesssim N \Phi_{2}(t)\left(\left\|\omega_{0}\right\|_{B_{\infty, 1}^{0}}+\left\|\frac{b^{\theta}}{r} b\right\|_{L_{t}^{1} B_{\infty, 1}^{1}}\right) .
\end{aligned}
$$

This, with Proposition 4.1f(ii), Proposition A.1f(ii), the embedding $B_{\sigma, 1}^{\frac{3}{\sigma}+1} \hookrightarrow B_{\infty, 1}^{1}$ and Proposition 4.3. yields

$$
\left\|\frac{b^{\theta}}{r} b\right\|_{L_{t}^{1} B_{\infty, 1}^{1}} \lesssim\left\|\frac{b^{\theta}}{r}\right\|_{L_{t}^{\infty} L^{\infty}}\|b\|_{L_{t}^{1} B_{\infty, 1}^{1}} \leqslant \Phi_{2}(t) .
$$

Now combining (6.9), (6.10) and (6.11) gives

$$
\|\omega(t)\|_{B_{\infty, 1}^{0}} \lesssim\left(2^{-N} e^{C V(t)}+\Phi_{2}(t) N\right)\left(\left\|\omega_{0}(t)\right\|_{B_{\infty, 1}^{0}}+\Phi_{2}(t)\right) .
$$

We choose $N$ such that

$$
N=[C V(t)]+1
$$

we obtain

$$
\|\omega(t)\|_{B_{\infty, 1}^{0}} \leqslant(V(t)+1) \Phi_{2}(t) .
$$

It remains to estimate $V(t)$. For this purpose we have by definition of Besov space, Bernstein inequality and the estimate $\left\|\Delta_{j} v\right\|_{L^{\infty}} \sim 2^{-j}\left\|\Delta_{j} \omega\right\|_{L^{\infty}}$,

$$
\begin{aligned}
\|v(t)\|_{B_{\infty, 1}^{1}} & =\sum_{j \geqslant-1} 2^{j}\left\|\Delta_{j} v(t)\right\|_{L^{\infty}} \\
& \lesssim\left\|\Delta_{-1} v(t)\right\|_{L^{\infty}}+\sum_{j \geqslant 0}\left\|\Delta_{j} \omega(t)\right\|_{L^{\infty}} \\
& \lesssim\|v(t)\|_{L^{\infty}}+\|\omega(t)\|_{B_{\infty, 1}^{0}} \\
& \leqslant \Phi_{3}(t)\left(1+\int_{0}^{t}\|v(\tau)\|_{B_{\infty, 1}^{1}} d \tau\right) .
\end{aligned}
$$

where we have used in the last inequality the estimate (6.13) and Proposition 4.4. Then, an application of the Gronwall inequality gives rise to

$$
\|v(t)\|_{B_{\infty, 1}^{1}} \lesssim \Phi_{4}(t) .
$$

Plugging this estimate into 6.13 gives,

$$
\|\omega(t)\|_{B_{\infty, 1}^{0}} \leqslant \Phi_{4}(t) .
$$

Using now the embeddings $B_{\infty, 1}^{1} \hookrightarrow \operatorname{Lip}\left(\mathbb{R}^{3}\right)$ and (6.14) we get

$$
\|\nabla v(t)\|_{L^{\infty}} \lesssim \Phi_{4}(t) .
$$

This concludes the proof of the proposition.

Now we will propagate the critical Besov $B_{p, 1}^{\frac{3}{p}+1}$ regularities globally in time for $2 \leqslant p<\infty$. More precisely, we prove the following proposition. 
Proposition 6.3. Let $\left(v_{0}, b_{0}\right) \in\left(L^{2} \cap B_{p, 1}^{\frac{3}{p}+1}\right) \times\left(L^{2} \cap B_{p, 1}^{\frac{3}{p}-1}\right)$ with $2 \leqslant p<\infty$. Assume, in addition that $\omega_{0}^{\theta} / r \in L^{3,1}$ and $b_{0}^{\theta} / r \in L^{2} \cap L^{\infty}$. Then for every $t \in \mathbb{R}_{+}$,

$$
\|v\|_{L_{t}^{\infty} B_{p, 1}^{1+\frac{3}{p}}}+\|b\|_{L_{t}^{\infty} B_{p, 1}^{\frac{3}{p}-1}}+\|b\|_{L_{t}^{1} B_{p, 1}^{\frac{3}{p}+1}} \leqslant \Phi_{6}(t) .
$$

Proof. We have by definition

$$
\begin{aligned}
\|v(t)\|_{B_{p, 1}^{1+\frac{3}{p}}} & =\sum_{j \geqslant-1} 2^{j\left(1+\frac{3}{p}\right)}\left\|\Delta_{j} v(t)\right\|_{L^{p}} \\
& \lesssim\left\|\Delta_{-1} v(t)\right\|_{L^{p}}+\sum_{j \geqslant 0} 2^{\frac{3}{p}}\left\|\Delta_{j} \omega(t)\right\|_{L^{p}} \\
& \lesssim\|v(t)\|_{L^{p}}+\|\omega(t)\|_{B_{p, 1}^{\frac{3}{p}}}
\end{aligned}
$$

We shall first estimate $\|\omega(t)\|_{B_{p, 1}^{\frac{3}{p}}}$. For this purpose we apply Proposition 2.3 to the vorticity equation (3.10), we obtain

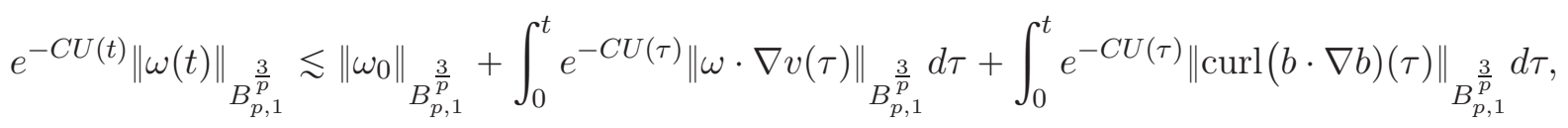

for all $p \in[1, \infty)$ where $U(t):=\|\nabla v\|_{L_{t}^{1} L^{\infty}}$. Since $\omega=\operatorname{curl} v$ then using Bony's decomposition we can show that (see page 36 from [3])

$$
\|\omega \cdot \nabla v(\tau)\|_{B_{p, 1}^{\frac{3}{p}}} \lesssim\|\omega\|_{B_{p, 1}^{\frac{3}{p}}}\|\nabla v(\tau)\|_{L^{\infty}} .
$$

Combining the two last estimates and using Gronwall's inequality we find

$$
\|\omega(t)\|_{B_{p, 1}^{\frac{3}{p}}} \lesssim\left(\left\|\omega_{0}\right\|_{B_{p, 1}^{\frac{3}{p}}}+\int_{0}^{t} e^{-C U(\tau)}\|\operatorname{curl}(b \cdot \nabla b)(\tau)\|_{B_{p, 1}^{\frac{3}{p}}} d \tau\right) e^{C U(t)} .
$$

Since $\operatorname{curl}(b \cdot \nabla b)=-\partial_{z}\left(\frac{b^{\theta}}{r} b\right)$ then using Proposition (A.1)-(ii) and Proposition (4.1)-(ii), we readily obtain

$$
\|\operatorname{curl}(b \cdot \nabla b)\|_{B_{p, 1}^{\frac{3}{p}}} \lesssim\left\|\frac{b^{\theta}}{r} b\right\|_{B_{p, 1}^{\frac{3}{p}+1}} \lesssim\left\|\frac{b^{\theta}}{r}\right\|_{L^{\infty}}\|b\|_{B_{p, 1}^{\frac{3}{p}+1}} \lesssim\left\|\frac{b_{0}^{\theta}}{r}\right\|_{L^{\infty}}\|b\|_{B_{p, 1}^{\frac{3}{p}+1}} .
$$

Combining the two last estimate, we find ,

$$
\|\omega(t)\|_{B_{p, 1}^{\frac{3}{p}}} \lesssim\left(\left\|\omega_{0}\right\|_{B_{p, 1}^{\frac{3}{p}}}+\left\|\frac{b_{0}^{\theta}}{r}\right\|_{L^{\infty}}\|b\|_{L_{t}^{1} B_{p, 1}^{\frac{3}{p}+1}}\right) e^{C U(t)} .
$$

Now we will estimate $\|v(t)\|_{L^{p}}$. Since the Riesz transforms act continuously on $L^{p}$, for all $1<p<\infty$, then from (4.23) we get

$$
\|v(t)\|_{L^{p}} \lesssim\left\|v_{0}\right\|_{L^{p}}+\int_{0}^{t}\|b \cdot \nabla b(\tau)\|_{L^{p}} d \tau+\int_{0}^{t}\|v(\tau)\|_{L^{p}}\|\nabla v(\tau)\|_{L^{\infty}} d \tau .
$$

Using Gronwall's inequality, the identity (3.9), the estimate (4.6) and the embedding $B_{p, 1}^{\frac{3}{p}+1} \hookrightarrow L^{p}$ we obtain, for all $p \in(1, \infty)$,

$$
\begin{aligned}
& \|v(t)\|_{L^{p}} \leqslant\left(\left\|v_{0}\right\|_{L^{p}}+\int_{0}^{t}\|b \cdot \nabla b(\tau)\|_{L^{p}} d \tau\right) e^{C\|\nabla v\|_{L_{t}^{1} L^{\infty}}} \\
& \lesssim\left(\left\|v_{0}\right\|_{L^{p}}+\left\|b^{\theta} / r\right\|_{L_{t}^{\infty} L^{\infty}}\|b\|_{L_{t}^{1} L^{p}}\right) e^{C\|\nabla v\|_{L_{t}^{1} L^{\infty}}}
\end{aligned}
$$

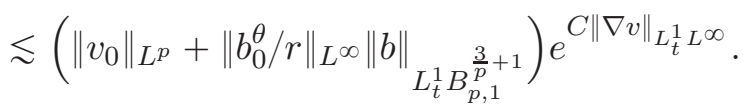


Inserting (6.17) and (6.18) into (6.16) gives

$$
\|v(t)\|_{B_{p, 1}^{1+\frac{3}{p}}} \lesssim\left(\left\|v_{0}\right\|_{L^{p}}+\left\|\omega_{0}\right\|_{B_{p, 1}^{\frac{3}{p}}}+\left\|\frac{b_{0}^{\theta}}{r}\right\|_{L^{\infty}}\|b\|_{L_{t}^{1} B_{p, 1}^{\frac{3}{p}+1}}\right) e^{C U(t)} .
$$

Now, to estimate the term $\|b\|_{L_{t}^{1} B_{p, 1}^{\frac{3}{p}+1}}$, we distinguish two cases:

Case $p>3$. Using the embedding $B_{\infty, 1}^{1} \hookrightarrow \operatorname{Lip}\left(\mathbb{R}^{3}\right)$, Proposition 4.3, and Proposition 6.2, we readily conclude that

$$
\|b\|_{L_{t}^{\infty} L^{p}}+\|b\|_{\widetilde{L}_{t}^{1} B_{p, 1}^{\frac{3}{p}+1}}+\|v(t)\|_{B_{p, 1}^{1+\frac{3}{p}}} \leqslant \Phi_{5}(t) .
$$

This achieves the proof for the case $p>3$.

Case $2 \leqslant p \leqslant 3$. In view of (4.16) we have

$$
\begin{gathered}
\|b\|_{\widetilde{L}_{t}^{\infty} B_{p, 1}^{\frac{3}{p}-1}}+\|b\|_{\widetilde{L}_{t}^{1} B_{p, 1}^{\frac{3}{p}+1}} \lesssim \\
+\sum_{q \geqslant 0} 2^{q\left(\frac{3}{p}-1\right)}\left\|\left[\Delta_{q}, v \cdot \nabla\right] b\right\|_{L_{t}^{1} L^{p}} .
\end{gathered}
$$

From Lemma 2.3, since $-1<\frac{3}{p}-1<\frac{1}{2}$ then one has

$$
\sum_{q \geqslant-1} 2^{q\left(\frac{3}{p}-1\right)}\left\|\left[\Delta_{q}, v \cdot \nabla\right] b\right\|_{L_{t}^{1} L^{p}} \lesssim \int_{0}^{t}\|\nabla v(\tau)\|_{L^{\infty}}\|b(\tau)\|_{B_{p, 1}^{\frac{3}{p}-1}} d \tau .
$$

In order to estimate the term $\|b \cdot \nabla v\|_{\widetilde{L}_{t}^{1} B_{p, 1}^{\frac{3}{p}-1}}$, in (6.20), we shall use the following product law

$$
\|b \cdot \nabla v\|_{B_{p, 1}^{\frac{3}{p}-1}} \lesssim \begin{cases}\|\nabla v\|_{L^{\infty}}\|b\|_{B_{p, 1}^{\frac{3}{p}-1}}+\|b\|_{L^{\infty}}\|v\|_{B_{p, 1}^{\frac{3}{p}}} & \text { if } \quad p<3 \\ \|v\|_{B_{\infty, 1}^{1}}\|b\|_{B_{p, 1}^{\frac{3}{p}-1}} & \text { if } \quad p=3\end{cases}
$$

where the first case follows from the fact that $B_{p, 1}^{\frac{3}{p}-1} \cap L^{\infty}$ is an algebra for every $p<3$ and the second one is proved in [25]. Thus, using the embeddings $B_{\infty, 1}^{1} \hookrightarrow \operatorname{Lip}\left(\mathbb{R}^{3}\right)$ and $B_{p, 1}^{\frac{3}{p}+1} \hookrightarrow B_{p, 1}^{\frac{3}{p}}$ we conclude that

$$
\|b \cdot \nabla v\|_{B_{p, 1}^{\frac{3}{p}-1}} \lesssim\|v\|_{B_{\infty, 1}^{1}}\|b\|_{B_{p, 1}^{\frac{3}{p}-1}}+\|b\|_{L^{\infty}}\|v\|_{B_{p, 1}^{\frac{3}{p}+1}} .
$$

Inserting (6.21) and (6.22) into (6.20) and using the embedding $B_{p, 1}^{\frac{3}{p}-1} \hookrightarrow L^{p}$ we obtain

$$
\|b\|_{\widetilde{L}_{t}^{\infty} B_{p, 1}^{\frac{3}{p}-1}}+\|b\|_{\widetilde{L}_{t}^{1} B_{p, 1}^{\frac{3}{p}+1}} \lesssim\left\|b_{0}\right\|_{B_{p, 1}^{\frac{3}{p}-1}}+\int_{0}^{t}\|b(\tau)\|_{L^{\infty}}\|v(\tau)\|_{B_{p, 1}^{\frac{3}{p}+1}} d \tau+\int_{0}^{t}\left(1+\|v(\tau)\|_{B_{\infty, 1}^{1}}\right)\|b(\tau)\|_{B_{p, 1}^{\frac{3}{p}-1}} d \tau .
$$

Then, Gronwall's inequality gives

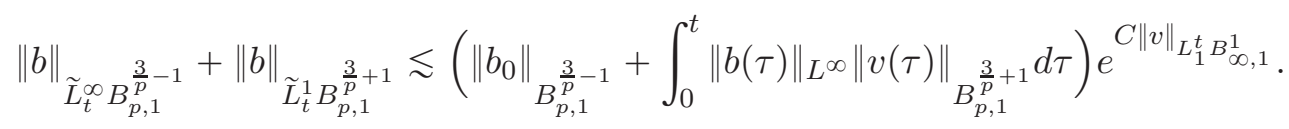


Plugging (6.23) into (6.19) we deduce that

$$
\|v(t)\|_{B_{p, 1}^{1+\frac{3}{p}}} \lesssim\left(\left\|v_{0}\right\|_{L^{p}}+\left\|\omega_{0}\right\|_{B_{p, 1}^{\frac{3}{p}}}+\left\|\frac{b_{0}^{\theta}}{r}\right\|_{L^{\infty}}\left\|b_{0}\right\|_{B_{p, 1}^{\frac{3}{p}-1}}+\left\|\frac{b_{0}^{\theta}}{r}\right\|_{L^{\infty}} \int_{0}^{t}\|b(\tau)\|_{L^{\infty}}\|v(\tau)\|_{B_{p, 1}^{\frac{3}{p}+1}} d \tau\right) e^{C\|v\|_{L_{1}^{t} B_{\infty, 1}^{1}}} .
$$

Applying again Granwall's inequality we get

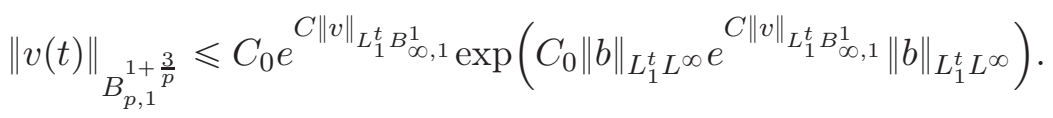

Finally, using the embedding $B_{p, 1}^{\frac{3}{p}-1} \hookrightarrow B_{\sigma, 1}^{\frac{3}{\sigma}-1}$, for some $\sigma>3$, Proposition 4.1 (iii) and Proposition 6.2 we conclude that

$$
\|v(t)\|_{B_{p, 1}^{1+\frac{3}{p}}} \leqslant \Phi_{6}(t)
$$

which give in turn, by (6.23),

$$
\|b\|_{\widetilde{L}_{t}^{\infty} B_{p, 1}^{\frac{3}{p}-1}}+\|b\|_{\widetilde{L}_{t}^{1} B_{p, 1}^{\frac{3}{p}+1}} \leqslant \Phi_{6}(t) .
$$

This achieves the proof of the Proposition.

\section{A Appendix}

Our task now is to prove the following product laws.

Proposition A.1. Let $b$ be a smooth axisymmetric vector field with a trivial radial component; $b^{r}=0$. Then the following estimate occurs

(i) For all $s>0$ and $t>0$ we have

$$
\left\|\frac{b^{\theta}}{r} b\right\|_{\widetilde{L}^{1} H^{s}} \leqslant C\left\|\frac{b^{\theta}}{r}\right\|_{L_{t}^{\infty} L^{\infty}}\|b\|_{\widetilde{L}^{1} H^{s}}
$$

(ii) For all $p \in[1, \infty]$ we have

$$
\left\|\frac{b^{\theta}}{r} b\right\|_{B_{p, 1}^{\frac{3}{p}+1}} \lesssim\left\|\frac{b^{\theta}}{r}\right\|_{L^{\infty}}\|b\|_{B_{p, 1}^{\frac{3}{p}+1}} .
$$

Proof. Using Bony's decomposition (2.2), we write

$$
\frac{b^{\theta}}{r} b=T_{\frac{b^{\theta}}{r}} b+T_{b} \frac{b^{\theta}}{r}+\mathcal{R}\left(\frac{b^{\theta}}{r}, b\right) .
$$

By definition of the paraproduct, we have

$$
T_{\frac{b^{\theta}}{r}} b=\sum_{k} S_{k-1}\left(\frac{b^{\theta}}{r}\right) \Delta_{k} b
$$

Then, by (2.1) for all $k \in \mathbb{N} \cup\{-1\}$ one has

$$
\Delta_{q}\left(T_{\frac{b^{\theta}}{r}} b\right)=\sum_{|k-q| \leqslant 4} \Delta_{q}\left(S_{k-1} \frac{b^{\theta}}{r} \Delta_{k} b\right) .
$$


Using Hölder inequality, we get

$$
\left\|\Delta_{q}\left(T_{\frac{b^{\theta}}{r}} b\right)\right\|_{L_{t}^{1} L^{2}} \leqslant\left\|\frac{b^{\theta}}{r}\right\|_{L_{t}^{\infty} L^{\infty}} \sum_{|k-q| \leqslant 4}\left\|\Delta_{q} b\right\|_{L_{t}^{1} L^{2}} .
$$

It follows that

$$
\left\|T_{\frac{b^{\theta}}{r}} b\right\|_{\widetilde{L}_{t}^{1} H^{s}}=\left\|2^{q s}\right\| \Delta_{q}\left(T_{\frac{b^{\theta}}{r}} b\right)\left\|_{L_{t}^{1} L^{2}}\right\|_{\ell^{2}} \lesssim\left\|\frac{b^{\theta}}{r}\right\|_{L_{t}^{\infty} L^{\infty}}\|b\|_{\widetilde{L}_{t}^{1} H^{s}} .
$$

For the remainder term we have by definition and by (2.1),

$$
\Delta_{j} \mathcal{R}\left(b, \frac{b^{\theta}}{r}\right)=\sum_{k \geqslant j-4} \Delta_{j}\left(\Delta_{k} b \widetilde{\Delta}_{k}\left(\frac{b^{\theta}}{r}\right)\right) .
$$

It follows that

$$
\left\|\Delta_{j} \mathcal{R}\left(b, \frac{b^{\theta}}{r}\right)\right\|_{L_{t}^{1} L^{2}} \lesssim \sum_{k \geqslant j-4}\left\|\Delta_{k} b\right\|_{L_{t}^{1} L^{2}}\left\|\widetilde{\Delta}_{k}\left(\frac{b^{\theta}}{r}\right)\right\|_{L_{t}^{\infty} L^{\infty}},
$$

where we have used the continuity of the operator $\Delta_{j}$ in $L^{2}$. Therefore

$$
\begin{aligned}
\left\|\left(2^{j s}\left\|_{\Delta_{j}} \mathcal{R}\left(b, \frac{b^{\theta}}{r}\right)\right\|_{L^{2}}\right)_{k}\right\|_{\ell^{2}} & \lesssim\left\|\frac{b^{\theta}}{r}\right\|_{L_{t}^{\infty} L^{\infty}}\left(\sum_{j \geqslant-1}\left(\sum_{k \geqslant j-4} 2^{(j-k) s} 2^{k s}\left\|\Delta_{k} b\right\|_{L_{t}^{1} L^{2}}\right)^{2}\right)^{\frac{1}{2}} \\
& \lesssim\left\|\frac{b^{\theta}}{r}\right\|_{L_{t}^{\infty} L^{\infty}}\|b\|_{\tilde{L}_{t}^{1} H^{s}} .
\end{aligned}
$$

To estimate the term $\left\|T_{b} \frac{b^{\theta}}{r}\right\|_{H^{s}}$, we use the axisymmetric structure of the vector field $b$. We write

$$
\Delta_{q}\left(T_{b} \frac{b^{\theta}}{r}\right)=\sum_{|k-q| \leqslant 4} \Delta_{q}\left(S_{k-1} b \Delta_{k}\left(\frac{b^{\theta}}{r}\right)\right) .
$$

Therefore, we have

$$
\left\|T_{b} \frac{b^{\theta}}{r}\right\|_{\widetilde{L}_{t}^{1} H^{s}} \lesssim\left\|\left(2^{k s}\left\|S_{k-1} b \Delta_{k}\left(\frac{b^{\theta}}{r}\right)\right\|_{L_{t}^{1} L^{2}}\right)_{k}\right\|_{\ell^{2}}
$$

Since $b^{r}=0$ then we can easily check that

$$
\frac{b^{\theta}}{r}=-\frac{b^{1}}{x_{2}}=\frac{b^{2}}{x_{1}}
$$

The term $S_{k-1} b \Delta_{k}\left(\frac{b^{\theta}}{r}\right)$ can be expanded under the form

$$
\begin{aligned}
S_{k-1} b^{1}(x) \Delta_{k}\left(\frac{b^{\theta}}{r}\right) & =-S_{k-1} b^{1}(x) \Delta_{k}\left(\frac{b^{1}}{x_{2}}\right) \\
& =-x_{2} S_{k-1}\left(\frac{b^{1}(x)}{x_{2}}\right) \Delta_{k}\left(\frac{b^{1}}{x_{2}}\right)+x_{2}\left[S_{k-1}, \frac{1}{x_{2}}\right] b^{1}(x) \Delta_{k}\left(\frac{b^{1}}{x_{2}}\right) \\
& =-S_{k-1}\left(\frac{b^{1}(x)}{x_{2}}\right) \Delta_{k} b^{1}-x_{2} S_{k-1}\left(\frac{b^{1}(x)}{x_{2}}\right)\left[\Delta_{k}, \frac{1}{x_{2}}\right] b^{1} \\
& +x_{2}\left[S_{k-1}, \frac{1}{x_{2}}\right] b^{1}(x) \Delta_{k}\left(\frac{b^{1}}{x_{2}}\right) \\
& :=-\mathrm{I}_{k}-\mathrm{II}_{k}+\mathrm{III}_{k} .
\end{aligned}
$$


For the first term, we immediately get

$$
\left\|\left(2^{k s}\left\|\mathrm{I}_{k}\right\|_{L_{t}^{1} L^{2}}\right)_{k}\right\|_{\ell^{2}} \lesssim\left\|\frac{b^{1}}{x_{2}}\right\|_{L_{t}^{\infty} L^{\infty}}\left\|b^{1}\right\|_{\widetilde{L}_{t}^{1} H^{s}}
$$

For the commutator term $\mathrm{II}_{k}$, we write by definition

$$
\begin{aligned}
\mathrm{II}_{k} & =S_{k-1}\left(\frac{b^{1}(x)}{x_{2}}\right) x_{2}\left(\Delta_{k}\left(\frac{b^{1}(x)}{x_{2}}\right)-\frac{\Delta_{k} b^{1}}{x_{2}}\right) \\
& =S_{k-1}\left(\frac{b^{1}(x)}{x_{2}}\right) 2^{3 k} \int_{\mathbb{R}^{3}} h\left(2^{k}(x-y)\right)\left(x_{2}-y_{2}\right) \frac{b^{1}}{y_{2}}(y) d y \\
& =2^{-k} S_{k-1}\left(\frac{b^{1}(x)}{x_{2}}\right)\left(2^{3 k} \tilde{h}\left(2^{k} .\right) *\left(\frac{b^{1}}{y_{2}}\right)(x)\right)
\end{aligned}
$$

where $\widetilde{h}(x)=x_{2} h(x)$. Since $\mathcal{F}(\widetilde{h}(\xi))=i \partial_{\xi_{2}} \mathcal{F}(h(\xi))=i \partial_{\xi_{2}} \varphi(\xi)$. Then it follows that Supp $\mathcal{F}(\tilde{h}) \subset$ Supp $\mathcal{F}(h)=\operatorname{Supp} \varphi$. Therefore for every $f \in \mathcal{S}^{\prime}$ we have

$$
2^{3 k} \tilde{h}\left(2^{k} .\right) * \Delta_{j} f=0, \text { for }|k-j| \geqslant 2 .
$$

This leads to

$$
2^{3 k} \tilde{h}\left(2^{k} .\right) * f=\sum_{|k-j| \leqslant 1} 2^{3 k} \widetilde{h}\left(2^{k} .\right) * \Delta_{j} f .
$$

Hence by Young inequality for convolution we get

$$
\begin{aligned}
\left\|\mathrm{II}_{k}\right\|_{L_{t}^{1} L^{2}} & \lesssim 2^{-k}\left\|S_{k-1}\left(\frac{b^{1}(x)}{x_{2}}\right)\right\|_{L_{t}^{\infty} L^{\infty}} \sum_{|k-j| \leqslant 1}\left\|2^{3 k} \tilde{h}\left(2^{k} .\right) * \Delta_{j}\left(\frac{b^{1}}{x_{2}}\right)\right\|_{L_{t}^{1} L^{2}} \\
& \lesssim 2^{-k}\|\widetilde{h}\|_{L^{1}}\left\|\frac{b^{1}}{x_{2}}\right\|_{L_{t}^{\infty} L^{\infty}} \sum_{|k-j| \leqslant 1}\left\|\Delta_{j}\left(\frac{b^{1}}{x_{2}}\right)\right\|_{L_{t}^{1} L^{2}} \cdot
\end{aligned}
$$

It follows that

$$
\begin{aligned}
\left\|\left(2^{k s}\left\|\mathrm{II}_{k}\right\|_{L_{t}^{1} L^{2}}\right)_{k}\right\|_{\ell^{2}} & \lesssim\left\|\frac{b^{1}}{x_{2}}\right\|_{L_{t}^{\infty} L^{\infty}}\left\|\left(2^{k(s-1)} \sum_{|k-j| \leqslant 1}\left\|\Delta_{j}\left(\frac{b^{1}}{x_{2}}\right)\right\|_{L_{t}^{1} L^{2}}\right)_{k}\right\|_{\ell^{2}} \\
& \lesssim\left\|\frac{b^{1}}{x_{2}}\right\|_{L_{t}^{\infty} L^{\infty}}\left\|\left(\sum_{|k-j| \leqslant 1} 2^{(k-j)(s-1)} 2^{j(s-1)}\left\|\Delta_{j}\left(\frac{b^{1}}{x_{2}}\right)\right\|_{L_{t}^{1} L^{2}}\right)_{k}\right\|_{\ell^{2}} \\
& \lesssim\left\|\frac{b^{1}}{x_{2}}\right\|_{L_{t}^{\infty} L^{\infty}}\left\|\frac{b^{1}}{x_{2}}\right\|_{\widetilde{L}_{t}^{1} H^{s-1}} .
\end{aligned}
$$

As for the term $\mathrm{III}_{k}$, we write by definition

$$
\begin{aligned}
\mathrm{III}_{k} & =\Delta_{k}\left(\frac{b^{1}(x)}{x_{2}}\right) x_{2}\left(S_{k-1}\left(\frac{b^{1}(x)}{x_{2}}\right)-\frac{S_{k-1} b^{1}(x)}{x_{2}}\right) \\
& =\Delta_{k}\left(\frac{b^{1}(x)}{x_{2}}\right) 2^{3(k-1)} \int_{\mathbb{R}^{3}} g\left(2^{(k-1)}(x-y)\right)\left(x_{2}-y_{2}\right) \frac{b^{1}}{y_{2}}(y) d y \\
& =2^{-k+1} \Delta_{k}\left(\frac{b^{1}(x)}{x_{2}}\right)\left(2^{3(k-1)} \widetilde{g}\left(2^{k-1} \cdot\right) *\left(\frac{b^{1}}{x_{2}}\right)(x)\right),
\end{aligned}
$$


where $\widetilde{g}(x)=x_{2} g(x)$. By Young inequality for convolution we get

$$
\begin{aligned}
\left\|\operatorname{III}_{k}\right\|_{L_{t}^{1} L^{2}} & \lesssim 2^{-k}\left\|\Delta_{k}\left(\frac{b^{1}}{x_{2}}\right)\right\|_{L_{t}^{1} L^{2}}\left\|2^{3 k} \widetilde{g}\left(2^{k} .\right) *\left(\frac{b^{1}}{x_{2}}\right)\right\|_{L_{t}^{\infty} L^{\infty}} \\
& \lesssim 2^{-k}\|\widetilde{g}\|_{L^{1}}\left\|\frac{b^{1}}{x_{2}}\right\|_{L_{t}^{\infty} L^{\infty}} 2^{-k}\left\|\Delta_{k}\left(\frac{b^{1}}{x_{2}}\right)\right\|_{L_{t}^{1} L^{2}} .
\end{aligned}
$$

This yields

$$
\begin{aligned}
\left\|\left(2^{k s}\left\|\mathrm{III}_{k}\right\|_{L_{t}^{1} L^{2}}\right)_{k}\right\|_{\ell^{2}} & \lesssim\left\|\frac{b^{1}}{x_{2}}\right\|_{L_{t}^{\infty} L^{\infty}}\left\|\left(2^{k(s-1)}\left\|\Delta_{k}\left(\frac{b^{1}}{x_{2}}\right)\right\|_{L_{t}^{1} L^{2}}\right)_{k}\right\|_{\ell^{2}} \\
& \lesssim\left\|\frac{b^{1}}{x_{2}}\right\|_{L_{t}^{\infty} L^{\infty}}\left\|\frac{b^{1}}{x_{2}}\right\|_{\tilde{L}_{t}^{1} H^{s-1}} .
\end{aligned}
$$

Thus, from (A.8) (A.9), (A.12) and (A.14) we deduce that

$$
\left\|\left(2^{k s}\left\|S_{k-1} b^{1} \Delta_{k}\left(\frac{b^{\theta}}{r}\right)\right\|_{L_{t}^{1} L^{2}}\right)_{k}\right\|_{\ell^{2}} \lesssim\left\|\frac{b^{1}}{x_{2}}\right\|_{L_{t}^{\infty} L^{\infty}}\left(\left\|\frac{b^{1}}{x_{2}}\right\|_{\tilde{L}_{t}^{1} H^{s-1}}+\left\|b^{1}\right\|_{\widetilde{L}_{t}^{1} H^{s}}\right) .
$$

Since $b$ is axisymmetric then $b^{1}\left(x_{1}, 0, z\right)=0$. Therefore from Taylor formula,

$$
b^{1}\left(x_{1}, x_{2}, z\right)=x_{2} \int_{0}^{1}\left(\partial_{x_{2}} b^{1}\right)\left(x_{1}, \tau x_{2}, z\right) d \tau .
$$

It follows that

$$
\begin{aligned}
\left\|\frac{b^{1}(x)}{x_{2}}\right\|_{\widetilde{L}_{t}^{1} H^{s-1}} & \lesssim \int_{0}^{1}\left\|\left(\partial_{x_{2}} b^{1}\right)(., \tau, .)\right\|_{\widetilde{L}_{t}^{1} H^{s-1}} d \tau \\
& \lesssim\left\|\partial_{x_{2}} b^{1}\right\|_{\widetilde{L}_{t}^{1} H^{s-1}} \int_{0}^{1} \tau^{s-\frac{5}{2}} d \tau \\
& \lesssim\left\|b^{1}\right\|_{\widetilde{L}_{t}^{1} H^{s}} .
\end{aligned}
$$

Inserting the last estimate into A.15 yields

$$
\left\|\left(2^{k s}\left\|S_{k-1} b^{1} \Delta_{k}\left(\frac{b^{\theta}}{r}\right)\right\|_{L_{t}^{1} L^{2}}\right)_{k}\right\|_{\ell^{2}} \lesssim\left\|\frac{b^{1}}{x_{2}}\right\|_{L_{t}^{\infty} L^{\infty}}\left\|b^{1}\right\|_{\tilde{L}_{t}^{1} H^{s}} .
$$

In a similar way we can show that

$$
\left\|\left(2^{k s}\left\|S_{k-1} b^{2} \Delta_{k}\left(\frac{b^{\theta}}{r}\right)\right\|_{L_{t}^{1} L^{2}}\right)_{k}\right\|_{\ell^{2}} \lesssim\left\|\frac{b^{2}}{x_{1}}\right\|_{L_{t}^{\infty} L^{\infty}}\left\|b^{2}\right\|_{\tilde{L}_{t}^{1} H^{s}} .
$$

Combining (A.16), A.17) and (A.7) we conclude that

$$
\left\|T_{b} \frac{b^{\theta}}{r}\right\|_{\widetilde{L}_{t}^{1} H^{s}} \lesssim\left\|\frac{b^{\theta}}{r}\right\|_{L_{t}^{\infty} L^{\infty}}\|b\|_{\widetilde{L}_{t}^{1} H^{s}} .
$$

Now putting together (A.7), A.6), A.18) and (A.1) we find

$$
\left\|\frac{b^{\theta}}{r} b\right\|_{\widetilde{L}_{t}^{1} H^{s}} \lesssim\left\|\frac{b^{\theta}}{r}\right\|_{L_{t}^{\infty} L^{\infty}}\|b\|_{\widetilde{L}_{t}^{1} H^{s}} .
$$

This concludes the proof of (i). 
To prove (ii) we use (A.1) to write

$$
\left\|\frac{b^{\theta}}{r} b\right\|_{B_{p, 1}^{\frac{3}{p}+1}} \leqslant\left\|T_{\frac{b^{\theta}}{r}} b\right\|_{B_{p, 1}^{\frac{3}{p}+1}}+\left\|T_{b} \frac{b^{\theta}}{r}\right\|_{B_{p, 1}^{\frac{3}{p}+1}}+\left\|\mathcal{R}\left(\frac{b^{\theta}}{r}, b\right)\right\|_{B_{p, 1}^{\frac{3}{p}+1}} .
$$

In view of (A.3) and by Hölder and Bernstein inequalities we get

$$
\begin{aligned}
\left\|T_{\frac{b^{\theta}}{r}} b\right\|_{B_{p, 1}^{\frac{3}{p}+1}} & \lesssim \sum_{q \in \mathbb{N}} 2^{q\left(\frac{3}{p}+1\right)}\left\|S_{q-1} \frac{b^{\theta}}{r} \Delta_{q} b\right\|_{L^{p}} \\
& \lesssim\left\|\frac{b^{\theta}}{r}\right\|_{L^{\infty}}\|b\|_{B_{p, 1}^{\frac{3}{p}+1}} .
\end{aligned}
$$

For the remainder term, by A.5), we have

$$
\begin{aligned}
\left\|\mathcal{R}\left(b, \frac{b^{\theta}}{r}\right)\right\|_{B_{p, 1}^{\frac{3}{p}+1}} & \lesssim \sum_{j \in \mathbb{N}} 2^{j\left(\frac{3}{p}+1\right)} \sum_{k \geqslant j-4}\left\|\Delta_{k} b\right\|_{L^{\infty}}\left\|\widetilde{\Delta}_{k}\left(\frac{b^{\theta}}{r}\right)\right\|_{L^{\infty}} \\
& \lesssim\left\|\frac{b^{\theta}}{r}\right\|_{L^{\infty}} \sum_{k} 2^{k\left(\frac{3}{p}+1\right)}\left\|\Delta_{k} b\right\|_{L^{\infty}} \sum_{j \leqslant k+4} 2^{(j-k)\left(\frac{3}{p}+1\right)} \\
& \lesssim\left\|\frac{b^{\theta}}{r}\right\|_{L^{\infty}}\|b\|_{B_{p, 1}^{\frac{3}{p}+1}}
\end{aligned}
$$

To estimate the term $\left\|T_{b} \frac{b^{\theta}}{r}\right\|_{B_{\infty, 1}^{1}}$, we write by definition

$$
\left\|T_{b} \frac{b^{\theta}}{r}\right\|_{B_{\infty, 1}^{1}} \lesssim \sum_{k \geqslant 0} 2^{k}\left\|S_{k-1} b(x) \Delta_{k}\left(\frac{b^{\theta}}{r}\right)\right\|_{L^{\infty}} .
$$

In view of (A.8), (A.10), (A.11) and (A.13) the term $S_{k-1} b^{1}(x) \Delta_{k}\left(\frac{b^{\theta}}{r}\right)$ can be decomposed as follow,

$$
S_{k-1} b^{1}(x) \Delta_{k}\left(\frac{b^{\theta}}{r}\right)=-\mathrm{I}_{k}-\mathrm{II}_{k}+\mathrm{III}_{k},
$$

with

$$
\begin{aligned}
\mathrm{I}_{k} & =S_{k-1}\left(\frac{b^{1}(x)}{x_{2}}\right) \Delta_{k} b^{1} \\
\mathrm{II}_{k} & =2^{-k} S_{k-1}\left(\frac{b^{1}(x)}{x_{2}}\right) \sum_{|k-q| \leqslant 1}\left(2^{3 k} \widetilde{h}\left(2^{k} .\right) * \Delta_{q}\left(\frac{b^{1}}{y_{2}}\right)(x)\right), \\
\mathrm{III}_{k} & =2^{-k+1} \Delta_{k}\left(\frac{b^{1}(x)}{x_{2}}\right)\left(2^{3(k-1)} \widetilde{g}\left(2^{k-1} .\right) *\left(\frac{b^{1}}{x_{2}}\right)(x)\right) .
\end{aligned}
$$

where $\widetilde{h}(x)=x_{2} h(x)$ and $\widetilde{g}(x)=x_{2} g(x)$. For the first term $\mathrm{I}_{k}$, we have

$$
\begin{aligned}
\sum_{k \geqslant 0} 2^{k\left(\frac{3}{p}+1\right)}\left\|\mathrm{I}_{k}\right\|_{L^{p}} & \lesssim\left\|\frac{b^{1}}{x_{2}}\right\|_{L^{\infty}} \sum_{k \geqslant 0} 2^{k}\left\|\Delta_{k} b^{1}\right\|_{L^{p}} \\
& \lesssim\left\|\frac{b^{1}}{x_{2}}\right\|_{L^{\infty}}\left\|b^{1}\right\|_{B_{p, 1}^{\frac{3}{p}+1}}
\end{aligned}
$$


For the second $\mathrm{II}_{k}$ and the third term $\mathrm{III}_{k}$, we shall make use of Young inequality for convolution,

$$
\begin{aligned}
\sum_{k \geqslant 0} 2^{k\left(\frac{3}{p}+1\right)}\left\|\mathrm{II}_{k}\right\|_{L^{p}} & \lesssim\left\|\frac{b^{1}}{x_{2}}\right\|_{L^{\infty}} \sum_{|k-q| \leqslant 1} 2^{q \frac{3}{p}}\|\widetilde{h}\|_{L^{1}}\left\|\Delta_{q}\left(\frac{b^{1}}{x_{2}}\right)\right\|_{L^{p}} \\
& \lesssim\left\|\frac{b^{1}}{x_{2}}\right\|_{L^{\infty}}\left\|\frac{b^{1}}{x_{2}}\right\|_{B_{p, 1}^{\frac{3}{p}}} \\
\sum_{k \geqslant 0} 2^{k\left(\frac{3}{p}+1\right)}\left\|\mathrm{III}_{k}\right\|_{L^{p}} & \lesssim\|\widetilde{g}\|_{L^{1}}\left\|\frac{b^{1}}{x_{2}}\right\|_{L^{\infty}} \sum_{k \geqslant 0} 2^{k \frac{3}{p}}\left\|_{k}\left(\frac{b^{1}}{x_{2}}\right)\right\|_{L^{p}} \\
& \lesssim\left\|\frac{b^{1}}{x_{2}}\right\|_{L^{\infty}}\left\|\frac{b^{1}}{x_{2}}\right\|_{B_{p, 1}^{\frac{3}{p}}}
\end{aligned}
$$

Thus, it follows from (A.24), (A.25) and (A.26) that

$$
\sum_{k \geqslant 0} 2^{k\left(\frac{3}{p}+1\right)}\left\|S_{k-1} b^{1}(x) \Delta_{k}\left(\frac{b^{\theta}}{r}\right)\right\|_{L^{p}} \lesssim\left\|\frac{b^{1}}{x_{2}}\right\|_{L^{\infty}}\left(\left\|\frac{b^{1}}{x_{2}}\right\|_{B_{p, 1}^{\frac{3}{p}}}+\left\|b^{1}\right\|_{B_{p, 1}^{\frac{3}{p}+1}}\right) .
$$

Since $b$ is axisymmetric then $b^{1}\left(x_{1}, 0, z\right)=0$. Therefore from Taylor formula,

$$
b^{1}\left(x_{1}, x_{2}, z\right)=x_{2} \int_{0}^{1}\left(\partial_{x_{2}} b^{1}\right)\left(x_{1}, \tau x_{2}, z\right) d \tau .
$$

For $1 \leqslant p<\infty$ one has

$$
\begin{aligned}
\left\|\frac{b^{1}(x)}{x_{2}}\right\|_{B_{p, 1}^{\frac{3}{p}}} & \lesssim\left\|\partial_{x_{2}} b^{1}\right\|_{B_{p, 1}^{\frac{3}{p}}} \\
& \lesssim\left\|b^{1}\right\|_{B_{\infty, 1}^{1}}
\end{aligned}
$$

For $p=\infty$ we use the estimate (6.8),

$$
\begin{aligned}
\left\|\frac{b^{1}(x)}{x_{2}}\right\|_{B_{\infty, 1}^{0}} & \lesssim\left\|\partial_{x_{2}} b^{1}\right\|_{B_{\infty, 1}^{0}} \int_{0}^{1}(1-\log \tau) d \tau \\
& \lesssim\left\|b^{1}\right\|_{B_{\infty, 1}^{1}} .
\end{aligned}
$$

Consequently,

$$
\sum_{k \geqslant 0} 2^{k\left(\frac{3}{p}+1\right)}\left\|S_{k-1} b^{1}(x) \Delta_{k}\left(\frac{b^{\theta}}{r}\right)\right\|_{L^{p}} \lesssim\left\|\frac{b^{1}}{x_{2}}\right\|_{L^{\infty}}\left\|b^{1}\right\|_{B_{p, 1}^{\frac{3}{p}+1}} .
$$

In a similar way we can prove the same estimate for the second component,

$$
\sum_{k \geqslant 0} 2^{k\left(\frac{3}{p}+1\right)}\left\|S_{k-1} b^{2}(x) \Delta_{k}\left(\frac{b^{\theta}}{r}\right)\right\|_{L^{p}} \lesssim\left\|\frac{b^{2}}{x_{1}}\right\|_{L^{\infty}}\left\|b^{2}\right\|_{B_{p, 1}^{\frac{3}{p}+1}} .
$$

It follows from (A.22), A.27) and (A.28) that

$$
\left\|T_{b} \frac{b^{\theta}}{r}\right\|_{B_{p, 1}^{\frac{3}{p}+1}} \lesssim\left\|\frac{b^{\theta}}{r}\right\|_{L^{\infty}}\|b\|_{B_{p, 1}^{\frac{3}{p}+1}} .
$$

Now putting together (A.19), (A.20), (A.21) and (A.29) we find

$$
\left\|\frac{b^{\theta}}{r} b\right\|_{B_{p, 1}^{\frac{3}{p}+1}} \lesssim\left\|\frac{b^{\theta}}{r}\right\|_{L^{\infty}}\|b\|_{B_{p, 1}^{\frac{3}{p}+1}} .
$$

This ends the proof of the proposition. 
Acknowledgments The author would like to thank T. Hmidi for the fruitful discussions about the critical case.

\section{References}

[1] G.V. Alexseev, Solvability of a homogeneous initial-boundary value problem for equations of magnetohydrodynamics of an ideal fluid, (Russian), Dinam. Sploshn. Sredy, 57 (1982), 3-20.

[2] H. Alfvén, Existence of electromagnetic-hydrodynamic waves, Nature, 150 (1942), 3805, 405-406.

[3] H. Abidi, T. Hmidi, and S. Keraani, S. On the global well-posedness for the axisymmetric Euler equations. Math. Ann. 347 (2010), 15-41

[4] H. Bahouri, J. -Y. Chemin and R. Danchin, Fourier Analysis and Nonlinear Partial Differential Equations, Grundlehren der Mathematischen Wissenschaften, 343 (2011).

[5] J. T. Beale, T. Kato and A. Majda, Remarks on the Breakdown of Smooth Solutions for the 3-D Euler Equations. Commun. Math. Phys. 94 (1984), p.61-66.

[6] D. Biskamp, Nonlinear Magnetohydrodynamics, Cambridge University Press, Cambridge, 1993.

[7] J.-M. Bony, Calcul symbolique et propagation des singularités pour les équations aux dérivées partielles non linéaires, Ann. de l'Ecole Norm. Sup., 14, (1981) p. 209-246, .

[8] J. Bergh, J. Löfström, Interpolation spaces. An introduction. Springer-Verlage, (1976).

[9] R. E. Caflisch, I. Klapper, and G. Steele. Remarks on singularities, dimension and energy dissipation for ideal hydrodynamics and MHD. Comm. Math. Phys., 184 (1997), 443-455.

[10] C. Cao and J. Wu, Global regularity for the 2D MHD equations with mixed partial dissipation and magnetic diffusion, Adv. Math., 226 (2011), 1803-1822.

[11] C. Cao, J. Wu and B. Yuan, The 2D incompressible magnetohydrodynamics equations with only magnetic diffusion SIAM J. Math. Anal. 46 (2014) 588-602.

[12] E. Casella; P. Secchi, P. Trebeschi. Global classical solutions for MHD system. J. Math. Fluid Mech. 5 (2003), 1, 70-91.

[13] S. Chandrasekhar. Hydrodynamic and hydromagnetic stability. The International Series of Monographs on Physics Clarendon Press, Oxford 1961

[14] J.-Y. Chemin, Perfect incompressible fluids. Oxford University Press (1998).

[15] J.-Y. Chemin, D. S. McCormick, J. C. Robinson and J. L. Rodrigo, Local existence for the non-resistive MHD equations in Besov spaces, Advances in Mathematics 286 (2016), 1-31

[16] Q. Chen, C. Miao, Z. Zhang. On the well-posedness of the ideal MHD equations in the Triebel-Lizorkin spaces. Arch. Ration. Mech. Anal. 195 (2010), 561-578.

[17] R. Danchin : Axisymmetric incompressible flows with bounded vorticity, Russian Math. Surveys 62 (2007), 73-94.

[18] R. Danchin, The inviscid limit for density-dependent incompressible fluids, Ann. Fac. Sci. Toulouse Math. 15 (2006), 637-688.

[19] P.A. Davidson, An Introduction to Magnetohydrodynamics, Cambridge Uni- versity Press, Cambridge, England, 2001.

[20] G. Duvaut and J.-L. Lions, Inéquations en thermoélasticité et magnéto- hydrodynamique, Arch. Rational Mech. Anal. 46 (1972), 241-279. 
[21] J. Fan, and T. Ozawa, , Regularity criteria for the magnetohydrodynamic equations with partial viscous terms and the Leray- $\alpha$-MHD model, Kinet. Relat. Models, 2(2009), 293-305.

[22] C. L. Fefferman, D. S. McCormick, J. C. Robinson and J. L. Rodrigo, Higher order commutator estimates and local existence for the non-resistive MHD equations and related models, J. Funct. Anal. 267 (2014) , 1035-1056.

[23] C. L. Fefferman, D. S. McCormick, J. C. Robinson and J. L. Rodrigo, Local existence for the non-resistive MHD equations in nearly optimal Sobolev spaces Arch. Rational Mech. Anal. 223 (2017), 677-691.

[24] T. Hmidi, The low Mach number limit for the isentropic Euler system with axisymmetric initial data. $J$. Inst. Math. Jussieu 12 (2013), 385-389.

[25] T. Hmidi and S. Keraani, Incompressible Viscous Flows in Borderline Besov Spaces. Arch Rational Mech Anal 189, (2008) 283-300

[26] T. Hmidi, S. Keraani, On the global well-posedness of the Boussinesq system with zero viscosity, Indiana Univ. Math. J. 58 (2009), 1591-1618.

[27] T. Hmidi, F. Rousset, Global well-posedness for the Euler-Boussinesq system with axisymmetric data, J. Funct. Anal. 260 (2011), 745-796.

[28] Q. Jiu, and D. Niu, Mathematical results related to a two-dimensional magneto-hydrodynamic equations, Acta Math. Sci. Ser. B Engl. Ed. 26 (2006), 744-756.

[29] Q. Jiu and J. Zhao, Global regularity of 2D generalized MHD equations with magnetic diffusion $Z$. Angew. Math. Phys. 66 (2015), 677-87.

[30] H. Kozono. Weak and classical solutions of the two-dimensional magnetohydrodynamic equations. Tohoku Math. J. 41 (1989), 471-488.

[31] Z. Lei, On axially symmetric incompressible magnetohydrodynamics in three dimensions J. Differential Equations 259 (2015), 3202-3215

[32] Z. Lei and Y. Zhou, BKM's criterion and global weak solutions for magnetohydrodynamics with zero viscosity, Discrete Contin. Dyn. Syst., 25 (2009), 575-583.

[33] P.-G. Lemarié, Recent Developments in the Navier-Stokes Problem, CRC Press, 2002.

[34] L.D. Laudau, E.M. Lifshitz, Electrodynamics of Continuous Media, 2nd ed., Pergamon, NewYork, 1984.

[35] C. Miao and B. Yuan, Well-posedness of the ideal MHD system in critical Besov spaces, Methods Appl. Anal. 13 (2006), 89-106.

[36] J. Peetre, New thoughts on Besov spaces, Duke University Mathematical Series 1, Durham N. C. (1976).

[37] P.G. Schmidt, On a magneto-hydrodynamic problem of Euler type, J. Diff. Eq., 74 (1988), 318-335.

[38] P. Secchi, On the equations of ideal incompressible magneto-hydrodynamics, Rend. Sem. Mat. Univ. Padova, 90 (1993), 103-119.

[39] Serfati, P.: Solutions $C^{\infty}$ en temps, $n$-log Lipschitz bornées en espace et équation d'Euler. C. R. Acad. Sci. Paris Sér. I Math. 320(1995), 555-558.

[40] M. Sermange and R. Temam, Some mathematical questions related to the MHD equations, Comm. Pure Appl. Math. 36 (1983), 635-664.

[41] S. Sulaiman On the global existence for the axisymmetric Euler-Boussinesq system in critical Besov space, Asymptot. Anal. 77 (2012), 89-121 
[42] T. Shirota and T. Yanagisawa, Note on global existence for axially symmetric solutions of the Euler system, Proc. Japan Acad. Ser. A Math. Sci., 70 (1994), 299-304.

[43] M. R. Uhkovskii, V. I. Iudovich, Axially symmetric flows of ideal and viscous fluids filling the whole space, Prikl. Mat. Meh. 32 (1968), 59-69.

[44] M. Vishik, Hydrodynamics in Besov spaces. Arch. Rational Mech. Anal., 145, (1998), 197-214.

[45] J. Wu. Viscous and inviscid magnetohydrodynamics equations. J. Anal. Math. 73 (1997), 251-265.

Z. Hassainia, NYU, Abu Dhabi Saadiyat Marina District - Abu Dhabi, United Arab Emirates. E-mail address: zh14@nyu.edu 